\title{
Self-interacting dark matter with scalar dilepton mediator
}

\author{
Chung Kao®, ${ }^{1, *}$ Yue-Lin Sming Tsai, ${ }^{2,3, \dagger}$ and Gwo-Guang Wong $\oplus^{4, \ddagger}$ \\ ${ }^{1}$ Homer L. Dodge Department of Physics, University of Oklahoma, Norman, Oklahoma 73019, USA \\ ${ }^{2}$ Key Laboratory of Dark Matter and Space Astronomy, Purple Mountain Observatory, \\ Chinese Academy of Sciences, Nanjing 210033, China \\ ${ }^{3}$ Department of Physics, National Tsing Hua University, Hsinchu 300, Taiwan \\ ${ }^{4}$ Department of Physics, National Taiwan University, Taipei 10617, Taiwan
}

(Received 31 December 2020; accepted 3 March 2021; published 29 March 2021)

\begin{abstract}
The cold dark matter (CDM) candidate with weakly interacting massive particles can successfully explain the observed dark matter relic density in cosmic scale and the large-scale structure of the Universe. However, a number of observations at the satellite galaxy scale seem to be inconsistent with CDM simulation. This is known as the small-scale problem of CDM. In recent years, it has been demonstrated that self-interacting dark matter (SIDM) with a light mediator offers a reasonable explanation for the smallscale problem. We adopt a simple model with SIDM and focus on the effects of Sommerfeld enhancement. In this model, the dark matter candidate is a leptonic scalar particle with a light mediator. We have found favored regions of the parameter space with proper masses and coupling strength generating a relic density that is consistent with the observed CDM relic density. Furthermore, this model satisfies the constraints of recent direct searches and indirect detection for dark matter as well as the effective number of neutrinos and the observed small-scale structure of the Universe. In addition, this model with the favored parameters can resolve the discrepancies between astrophysical observations and $N$-body simulations.
\end{abstract}

DOI: 10.1103/PhysRevD.103.055021

\section{INTRODUCTION}

The first evidence of dark matter (DM) was observed by Fritz Zwicky [1] in 1933. The existence of dark matter can be observed in the whole Universe, at the small galactic scale $[2,3]$, the large scale of galaxy clusters [4,5], and the cosmological scale [6,7]. The evidence of dark matter is usually inferred from its gravitational interactions. However, weakly interacting massive particles (WIMPs) provide intuitive candidates as cold dark matter (CDM). Stable invisible WIMPs with proper mass and coupling strength can lead to a matter density that is consistent with the observed DM relic density in cosmic scale structure of the Universe [8]. In addition, CDM can account for the consistency of large scale structure $(\gtrsim 1 \mathrm{Mpc})$ in the Universe between the astrophysical observations [9] and $N$-body simulations [10].

There exist some discrepancies between CDM $N$-body simulations and astrophysical observations on small scale

\footnotetext{
*Chung.Kao@ou.edu

†smingtsai@pmo.ac.cn

ggwong@hep1.phys.ntu.edu.tw
}

Published by the American Physical Society under the terms of the Creative Commons Attribution 4.0 International license. Further distribution of this work must maintain attribution to the author(s) and the published article's title, journal citation, and DOI. Funded by SCOAP. structure of the Universe. The first one is the cusp-core problem (CCP) [11]. The observed mass distributions are more flat in the central region of dwarf galaxies without a steep cusp predicted from CDM simulations. The second one is the missing satellite problem (MSP) [12]. The observed number of dwarf satellite galaxies in the Milky Way (MW) is much less than that predicted from CDM simulations. In recent years there is another problem originally from the MSP, which is called the too-big-to-fail (TBTF) $[13,14]$. Most massive subhalos generated from the CDM $N$-body simulation are too massive in the Milky Way halo with circular velocity larger than $30 \mathrm{~km} / \mathrm{s}$, whereas the observed maximum circular velocities of dwarf spheroidals are less than $25 \mathrm{~km} / \mathrm{s}$.

All three problems, CCP, MSP, and TBTF, are called the small scale problem and they can be resolved if the CDM particles are self-interacting with a light mediator to give a large self-interacting cross section (SICS) [15]. The large SICS provides a positive gradient of velocity dispersion / temperature near the center of (sub)halo such that the heat flow moves outward avoiding the formation of a density cusp until self-interaction becomes weak and forms a flat core density. Also, the smaller (sub)halo has a lower temperature since the faster DM particles are easily to escape from the long-range gravitational potential. Hence, with the smaller subhalo in the host halo, the large SICS can transfer heat from hotter DM particles in host halo to 
the colder DM particle in subhalo resulting in the subhalo's fragmentation or evaporation. On the other hand, the selfinteracting cross section can not be too large. Otherwise, the small structures of our Universe such as satellite galaxies would likely be washed out.

From the astrophysical observation of galaxies and clusters of galaxy, we have the following constraints.

(i) For a galaxy cluster with circular velocity $v \simeq$ $1000 \mathrm{~km} / \mathrm{s}$, the momentum transfer cross section $\left(\sigma_{T}\right)$ per unit DM mass is $\sigma_{T} / m_{\chi} \simeq 0.1 \mathrm{~cm}^{2} / \mathrm{g}$ [16].

(ii) For a galaxy with $v \simeq 100 \mathrm{~km} / \mathrm{s}, \sigma_{T} / m_{\chi} \simeq 2 \mathrm{~cm}^{2} / \mathrm{g}$.

(iii) For a dwarf galaxy with $v \simeq 10-100 \mathrm{~km} / \mathrm{s}, \sigma_{T} / m_{\chi} \simeq$ $5-10 \mathrm{~cm}^{2} / \mathrm{g}$ [17].

We can see that the SICS increases with decreasing DM velocity. The velocity-dependent SICS is required to solve the problems occurred in small structure scale of Universe. In addition, possible anomalies in cosmic-ray, positron excess observed by PAMELA [18], AMS02 [19], ATIC [20] and FermiLAT [21] can also be explained by requiring that the present DM annihilation cross section be $2 \sim 3$ orders of magnitude greater than that in the freeze-out stage [22]. This can be achieved by considering the Sommerfeld effect on a SIDM with a light mediator and can also be constrained by these anomalous observations of cosmic-ray [23].

A simple and elegant model with a self-interacting leptonic scalar dark matter $(\chi)$ and a light mediator $(\zeta)$ was recently proposed [24] to provide a CDM candidate and to solve the small scale problems of the Universe. The light mediator $(\zeta)$ could have a large production cross section through s-wave Sommerfeld enhancement at late times. If it decays to electrons and photons, it would change the history of gas ionization in the Universe, disrupt the cosmic microwave background (CMB), and be ruled out by the precise cosmological data now available [25]. To satisfy cosmological requirements, the mediator $(\zeta)$ is chosen to have special Yukawa couplings such that it would not decay into electrons and photons [24]. In our analysis, we have adopted this model with a focus on Sommerfeld enhancement to determine the CDM relic density more precisely. In addition, we find the allowed parameter space that satisfies all constraints of (a) recent direct searches (b) indirect detection experiments, (c) the observed relic density, (d) effective number of neutrinos, and (e) the astrophysical observation of small-scale structure of Universe.

This paper is organized as follows. In Sec. II, we introduce a leptonic scalar dark matter (LSDM) model proposed by E. Ma [24]. Section III shows direct search results for leptonic scalar dark matter, the spin-independent cross section of DM-nucleon elastic scattering in the LSDM model, and compare it with XENON1T data [26]. In Sec. IV, we present DM relic density as well as discovery potential of indirect search for CDM. We evaluate the relic density and effects of the Sommerfeld enhancement in the LSDM model to compare with the observed DM relic density [8,27]. In addition, we compare our results of indirect search with Fermi-LAT $[28,29]$ and H.E.S.S. [30] astrophysical observations. The cosmological constraints on right-handed neutrino and the small scale requirements (CCP, MSP, TBTF) are discussed in Secs. V and VI, respectively. We show favored regions of parameter space in Sec. VII. Conclusions are drawn in Sec. VIII. Some useful formulas for Sommerfeld enhancement are presented in Appendix A.

\section{LEPTONIC SCALAR DARK MATTER MODEL}

Recently, a simple and elegant model with a selfinteracting leptonic scalar dark matter (LSDM) was proposed [24]. This model is a simple extension of the Standard Model (SM) with conservation of a $U(1)_{\mathrm{L}}$ lepton number. There exist a singlet scalar $(\chi)$ chosen to be the DM candidate with $L=1$, one light singlet scalar $(\zeta)$ as a mediator with $L=2$, and three right-handed neutrinos: $\nu_{R i}$ $(i=1,2,3)$. The lepton number conservation assures the stability of $\chi$ and no vacuum expectation value developed from $\chi$ and $\zeta$ scalar fields.

The general scalar potential consisting of $\chi, \zeta$ and the SM Higgs doublet is given by [24]

$$
\begin{aligned}
V= & \mu_{0}^{2} \Phi^{\dagger} \Phi+\mu_{1}^{2} \chi^{*} \chi+\mu_{2}^{2} \zeta^{*} \zeta+\left(\mu_{12} \zeta^{*} \chi^{2}+\text { H.c. }\right) \\
& +\frac{1}{2} \lambda_{0}\left(\Phi^{\dagger} \Phi\right)^{2}+\frac{1}{2} \lambda_{1}\left(\chi^{*} \chi\right)^{2}+\frac{1}{2} \lambda_{2}\left(\zeta^{*} \zeta\right)^{2} \\
& +\lambda_{01}\left(\Phi^{\dagger} \Phi\right)\left(\chi^{*} \chi\right)+\lambda_{02}\left(\Phi^{\dagger} \Phi\right)\left(\zeta^{*} \zeta\right)+\lambda_{12}\left(\chi^{*} \chi\right)\left(\zeta^{*} \zeta\right) .
\end{aligned}
$$

The scalar masses have the following relations

$$
\begin{aligned}
& m_{H}^{2}=\lambda_{0} \mathrm{v}^{2} \simeq(125 \mathrm{GeV})^{2}, \quad m_{\chi}^{2}=\mu_{1}^{2}+\frac{1}{2} \lambda_{01} \mathrm{v}^{2}, \\
& m_{\zeta}^{2}=\mu_{2}^{2}+\frac{1}{2} \lambda_{02} \mathrm{v}^{2},
\end{aligned}
$$

and the Higgs vacuum expectation value is $\mathrm{v} \simeq 246 \mathrm{GeV}$.

For simplicity, let us consider a $C P$-conserving scalar potential with eight free real parameters:

$$
m_{\chi}, m_{\zeta}, \mu_{12}, \lambda_{1}, \lambda_{2}, \lambda_{01}, \lambda_{02}, \quad \text { and } \lambda_{12} \text {. }
$$

The values of $\mu_{0}$ and $\lambda_{0}$ are fixed by the minimization condition of the scalar potential and the measured Higgs mass. The $\mu_{12} \zeta^{*} \chi^{2}$ term serves as the source to enhance the self-interaction of $\chi \chi^{*} \rightarrow \chi \chi^{*}$ through the exchange of $\zeta$. That leads to the dominant $t$-channel cross section

$$
\sigma\left(\chi \chi^{*} \rightarrow \chi \chi^{*}\right)=\frac{\mu_{12}^{4}}{4 \pi m_{\zeta}^{4} m_{\chi}^{2}},
$$

where $\zeta$ is the light mediator [24]. 


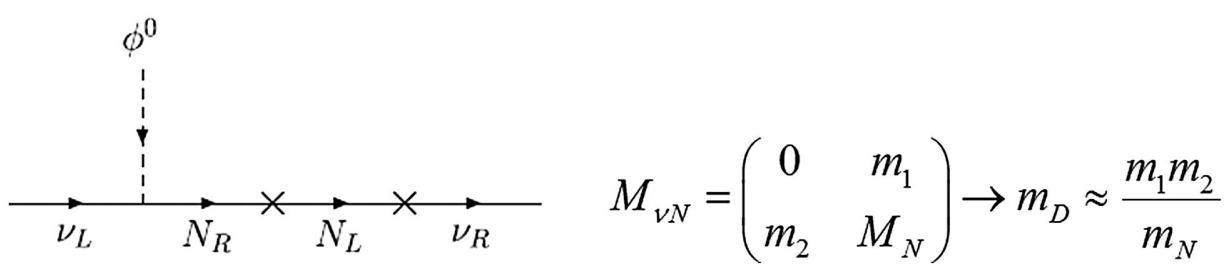

FIG. 1. Dirac neutrino mass with a Dirac singlet fermion insertion.

The neutrinos in this model are Dirac fermions with small masses that could be natural consequences of various known mechanisms [31-33]. For example, let us consider a discrete symmetry $S$, such that under a transformation we have

$$
\nu_{L} \rightarrow+\nu_{L}, \quad \phi^{0} \rightarrow+\phi^{0}, \quad \text { and } \quad \nu_{R} \rightarrow-\nu_{R},
$$

Then we insert a heavy singlet Dirac fermion $N$ with a large mass $M_{N}$ as shown in Fig. 1 . The $\mathrm{S}$ symmetry is softly broken by the dimension three mass term $\bar{\nu}_{R} N_{L}$ with $N \rightarrow+N$ and $\nu_{R} \rightarrow-\nu_{R}$. The small masses $m_{1}$ and $m_{2}$ are generated by electroweak symmetry breaking or soft $S$ symmetry breaking. That leads to a small Dirac neutrino mass through the seesaw mechanism. Thus, the only new Yukawa couplings are

$$
\mathcal{L}_{Y}=f_{i j} \zeta^{*} \overline{\nu_{R}^{c}} \nu_{R}+\text { H.c.. }
$$

After $\chi$ freezes out, $\zeta$ eventually decays to neutrinos via the $f_{i j}$ terms with a lifetime

$$
\tau_{\zeta}=\frac{4 \pi}{m_{\zeta} f_{R R}^{2}},
$$

where $f_{R R}^{2} \equiv \sum_{i, j}\left|f_{i j}\right|^{2}$. We can see that $\tau_{\zeta} \lesssim 10^{-11} \mathrm{sec}$, for $m_{\zeta}>m_{\zeta}^{\min }=0.2 \mathrm{GeV}$ and $f_{R R}>f_{R R}^{\min }=10^{-6}$. This means that before the onset of big bang nucleosynthesis (BBN), all $\zeta$ 's decay away and $\nu_{R}$ decouples from the SM particles at the temperature $T_{f}^{R} \simeq m_{\zeta}$.

Before going further, we should note that $\chi^{*}$ has a lepton number $L=-1$ and it is the antiparticle of $\chi$ with $L=+1$. Since $C P$ is conserved, the transition amplitude $\left(M_{\chi^{*}}\right)$ involving $\chi^{*}$ is the same as the transition amplitude $\left(M_{\chi}\right)$ with $\chi$. Now let $n_{\chi}$ and $n_{\chi^{*}}$ be the number density of the particle $\chi$ and the antiparticle $\chi^{*}$, respectively. Their sum is total number density of DM, $n=n_{\chi}+n_{\chi^{*}}$. Both $\chi$ and $\chi^{*}$ make equal amount of contributions to the annihilation cross section and the CDM relic density.

\section{DIRECT SEARCH FOR LEPTONIC SCALAR DARK MATTER}

It is an opportune time to investigate direct detection for WIMP dark matter. The XENON1T collaboration recently announced the observation of low energy excess electronic recoil events in their detector [34]. In this section, we focus on the search for nuclear recoils generated by the WIMPnucleon scattering. We evaluate $\chi$-nucleon elastic scattering cross section for the leptonic scalar dark matter $(\chi)$. In addition, we place limits on the relevant parameters $\lambda_{01}$ and $m_{\chi}$ with XENON1T results.

\section{A. The elastic scattering of DM with nucleus $\mathcal{N}$}

In the LSDM model, the leptonic scalar DM $(\chi)$ interacts with quarks $(q)$ through the SM Higgs boson $(H)$. Hence the effective Lagrangian of $\chi$ with quarks is simply

$$
\mathcal{L}_{\text {eff }}=\sum_{q} a_{q}\left[\chi \chi^{*}\right][\bar{q} q], \quad \text { with } \quad a_{q} \simeq \lambda_{01} \frac{m_{q}}{m_{H}^{2}},
$$

where $m_{q}$ and $m_{H}$ are masses of the quark and the Higgs boson, respectively, and $a_{q}$ is the effective coupling of DM interacting with the quark in a nucleon.

Before making an estimate for the detection rate of the leptonic dark matter $(\chi)$ in the XENON1T experiment, let us evaluate the normalized spin independent (SI) cross section for the leptonic scalar DM scattered off the ${ }^{129,131} \mathrm{Xe}$ nuclei. Applying the effective Lagrangian in Eq. (8), we obtain the matrix element for elastic scattering of the DM particle $(\chi)$ and the nucleus $(\mathcal{N})$ as

$$
M_{f i}=2 m_{\mathcal{N}} \sum_{q} a_{q}\left\langle\mathcal{N}_{f}|\bar{q} q| \mathcal{N}\right\rangle
$$

In general, the averaged unpolarized amplitude square at $q^{2}=0$ can be written as

$$
\begin{aligned}
& \bar{\sum}\left|M_{f i}\right|^{2}\left(q^{2}=0\right) \\
& \quad=\sum\left|M_{f i}^{\mathrm{SI}}\right|^{2}\left(q^{2}=0\right)+\bar{\sum}\left|M_{f i}^{\mathrm{SD}}\right|^{2}\left(q^{2}=0\right) \\
& \quad=4 m_{\mathcal{N}}^{2} f_{s \mathcal{N}}^{2},
\end{aligned}
$$

where SI and SD denote the spin independent and the spin dependent contributions, respectively.

For the effective scalar interaction, we have

$$
f_{s \mathcal{N}}=Z f_{s p}+(A-Z) f_{s n},
$$

where 


$$
\begin{aligned}
f_{s p(n)}= & \sum_{q=u, d, s} a_{q} \frac{m_{p(n)}}{m_{q}} f_{T q}^{p(n)} \\
& +\sum_{q=c, b, t} a_{q} \frac{2}{27} \frac{m_{p(n)}}{m_{q}}\left(1-\sum_{q^{\prime}=u, d, s} f_{T q^{\prime}}^{p(n)}\right) .
\end{aligned}
$$

When evaluating the quark operator matrix element in the nuclear state, we need to include loop contributions involving heavy quarks that contribute to the mass of the nucleon $\left(m_{p(n)}\right)$.

The proton mass fraction $f_{T q}^{p}$ is defined by the matrix elements of the quark current

$$
\left\langle p\left|m_{q} \bar{q} q\right| p\right\rangle= \begin{cases}m_{p} f_{T q}^{p}, & q=u, d, s, \\ \frac{2}{27} m_{p}\left(1-\sum_{q=u, d, s} f_{T q}^{p}\right), & q=c, b, t\end{cases}
$$

The neutron mass fraction $f_{T q}^{n}$ is defined in the same way. The matrix elements of the light-quark currents in the proton or neutron are obtained in chiral perturbation theory from measurements of the pion-nucleon sigma term [35-40]. The heavy quark contribution to the mass of the nucleon is through the triangle diagram [41].

In the center of mass $(\mathrm{CM})$ frame, the differential cross section is

$$
\frac{d \sigma(\vec{q}=0)}{d|\mathbf{q}|^{2}}=\frac{1}{64 \pi s \mu_{\mathcal{N}}^{2} v^{2}} \sum\left|M_{f i}\right|^{2}\left(q^{2}=0\right),
$$

where $v$ is the DM velocity relative to the target, $\sqrt{s} \approx$ $m_{\chi}+m_{\mathcal{N}}$ is the total energy, and $\mu_{\mathcal{N}}$ is the reduced mass of $\mathrm{DM}$ and the target nucleus $\mathcal{N}$. The total cross section at zero momentum transfer [42] can then be obtained as

$$
\sigma_{0}^{S I}=\int_{0}^{4 \mu_{\mathcal{N}}^{2} v^{2}} d|\mathbf{q}|^{2} \frac{d \sigma(\vec{q}=0)}{d|\mathbf{q}|^{2}}=\frac{\mu_{\mathcal{N}}^{2}}{\pi} f_{s \mathcal{N}}^{2}
$$

Hence the total cross section of DM-nucleus $(\chi-\mathcal{N})$ scattering becomes

$$
\sigma_{\mathcal{N}}=\frac{\sigma_{0}^{\mathrm{SI}}}{4 \mu_{\mathcal{N}}^{2} v^{2}} \int_{0}^{4 \mu_{\mathcal{N}}^{2} v^{2}} d|\mathbf{q}|^{2} F_{\mathrm{SI}}^{2}(|\mathbf{q}|)
$$

where $F_{\mathrm{SI}}^{2}(|\mathbf{q}|)$ is the spin-independent form factor. To compare with the experimental results, we define the scaled SI and SD cross sections, respectively, for the nucleus with atomic mass number $A_{i}$ and isotope abundance $\eta_{i}$ as the following

$$
\sigma_{\chi p}^{\mathrm{SI}} \equiv \frac{\sum_{i} \eta_{i} \sigma_{A_{i}}}{\sum_{j} \eta_{j} A_{j}^{2} \frac{\mu_{A_{j}}^{2}}{\mu_{p}^{2}}},
$$

and

$\sigma_{\chi p, n}^{\mathrm{SD}} \equiv\left(\sum_{i} \eta_{i} \sigma_{A_{i}}\right)\left(\sum_{j} \eta_{j} \frac{4 \mu_{A_{j}}^{2}\left\langle S_{p, n}\right\rangle_{\mathrm{eff}}^{2}\left(J_{A_{j}}+1\right)}{3 \mu_{p, n}^{2} J_{A_{j}}}\right)^{-1}$,

where $\mu_{A_{i}}$ and $\mu_{p, n}$ are the reduced masses of the DM with the target nucleus and the DM with proton or neutron, respectively. In the above, $\left\langle S_{p(n)}\right\rangle_{\text {eff }}$ and $\left(J_{A_{j}}\right)$ are the proton (neutron) spin expectation value (including the contributions of two-body current [43]) and the total angular momentum of the nucleus with atomic mass number $A_{j}$ respectively. The effective spin expectation value is defined as $\left\langle S_{p(n)}\right\rangle_{\text {eff }} \equiv\left\langle S_{p(n)}\right\rangle \pm \delta a_{1}\left(\left\langle S_{p}\right\rangle-\left\langle S_{n}\right\rangle\right) / 2$ and $\delta a_{1}$ is the fraction contributing to the isovector coupling $[43,44]$.

\section{B. Numerical results for direct search}

At present, the XENON1T experiment [26] provides the most stringent upper limits on $\sigma^{\text {SI }}$ for WIMP masses above $6 \mathrm{GeV}$. In our analysis for spin independent cross section of $\chi-\mathcal{N}$ scattering, we adopt the Helm form factor [45,46] used in XENON1T experiments:

$$
F_{\mathrm{SI}}^{2}(|\mathbf{q}|)=\left(\frac{3 j_{1}\left(q R_{\mathcal{N}}\right)}{q R_{\mathcal{N}}}\right)^{2} e^{(q s)^{2}}
$$

where the nuclear radius $R_{\mathcal{N}}=c^{2}+\frac{7}{3} \pi^{2} a^{2}-5 s^{2}$ with $c=\left(1.23 A^{1 / 3}-0.6\right) \mathrm{fm}, \quad a=0.52 \mathrm{fm}$ and the nuclear surface thickness $s=1 \mathrm{fm}$. We use the updated data of nucleon mass fractions from Ref. [40]: $f_{T u}^{p}=0.017$, $f_{T d}^{p}=0.023, f_{T u}^{n}=0.012, f_{T d}^{n}=0.033, f_{T s}^{p, n}=0.053$.

In the LSDM model with a scalar dark matter $(\chi)$ and a light mediator $(\zeta)$, there are eight free parameters as shown in Eq. (3). In our analysis, the scan is performed with the log-prior distributions for the input parameters as shown in the below:

(i) $m_{H} / 2 \leq m_{\chi} \leq 1 \mathrm{TeV}$, such that $\chi \chi \rightarrow \zeta H$ can occur,

(ii) $0.2 \mathrm{GeV} \leq m_{\zeta} \leq 1.2 \mathrm{GeV}$,

(iii) $1 \mathrm{GeV} \leq \mu_{12} \leq 1 \mathrm{TeV}$, and

(iv) $10^{-6} \leq \lambda \leq \mathcal{O}(1) \sim \sqrt{4 \pi}$ for $\lambda=\lambda_{1}, \lambda_{2}, \lambda_{01}$, or $\lambda_{12}$. Note that $\lambda_{02}$ is chosen to be $10^{-6} \leq \lambda_{02} \leq 10^{-2}$. It is constrained by the SM Higgs invisible decay width $\left(H \rightarrow \zeta \zeta^{*}\right)$, i.e.,

$$
\Gamma\left(H \rightarrow \zeta \zeta^{*}\right)=\frac{\lambda_{02}^{2} v^{2}}{16 \pi m_{H}} .
$$




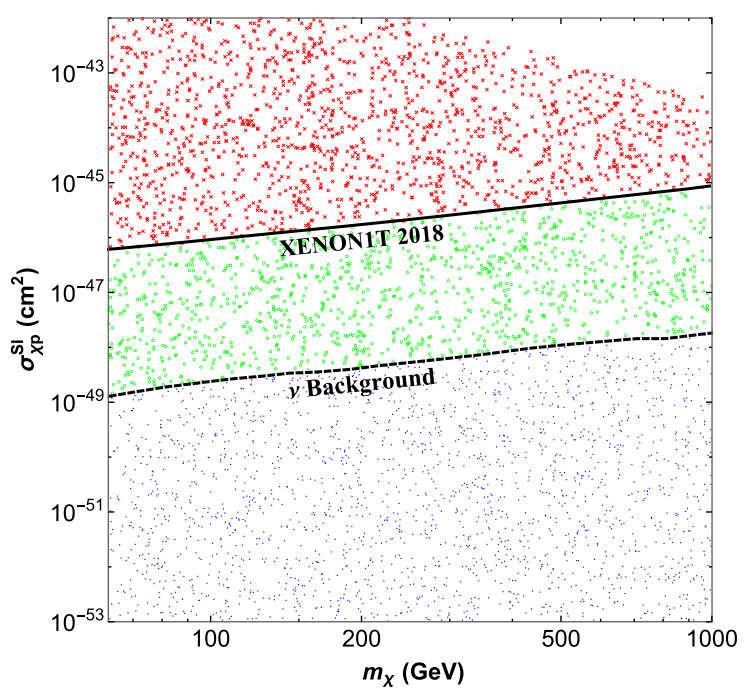

(a)

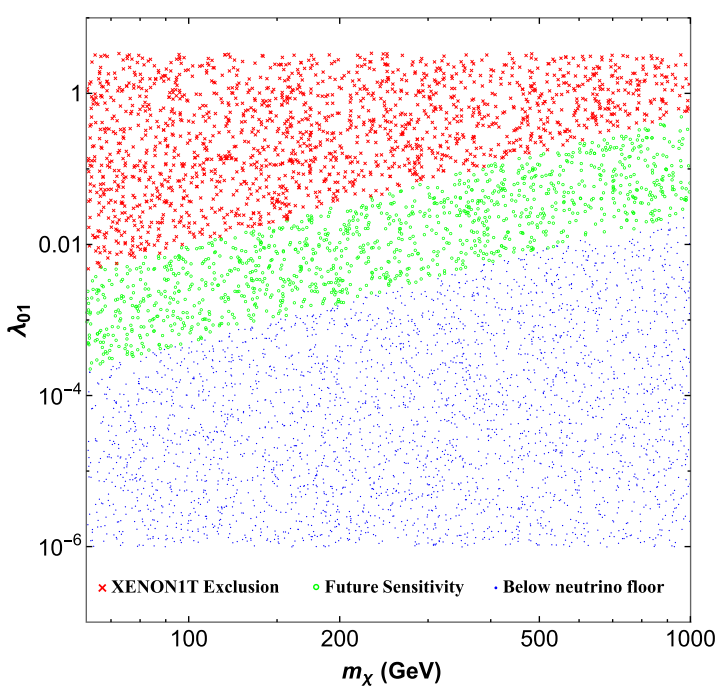

(b)

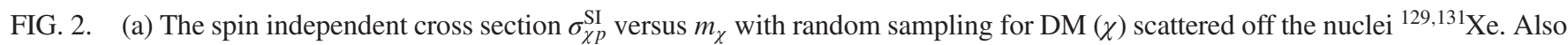
shown are the upper limit from XENON1T [26] and the neutrino background [47]. (b) A scatter plot for the same samples projected to the plane of $\left(m_{\chi}, \lambda_{01}\right)$ with the corresponding $\lambda_{01}$.

Assuming that the invisible width is less than $10 \%$ of the Higgs width $\Gamma_{H} \sim 4.12 \mathrm{MeV}$ [27], we obtain the maximal value of $\lambda_{02} \sim 6.5 \times 10^{-3}$. In addition, $m_{\zeta}$ must be greater than $0.2 \mathrm{GeV}$ to satisfy the cosmological constraint of effective number of neutrinos, which will be discussed later.

In this model, the scaled SI and SD cross sections $\left(\sigma_{\chi p}^{\mathrm{SI}}\right.$ and $\sigma_{\chi p}^{\mathrm{SD}}$ ) depend on two parameters: (i) the mass of leptonic scalar dark matter $\left(m_{\chi}\right)$, and (ii) the coupling $\lambda_{01}$. We apply the most stringent constraint from XENON1T experiment [26] with the upper limits of SI WIMP-nucleon scattering cross section. Figure 2(a) shows the spin independent cross section $\sigma_{\chi p}^{\text {SI }}$ versus $m_{\chi}$ with random sampling for DM $(\chi)$ scattered off the nuclei ${ }^{129,131} \mathrm{Xe}$. In addition, a scatter plot for the same samples projected to the plane of $\left(m_{\chi}, \lambda_{01}\right)$ with the corresponding $\lambda_{01}$ is presented in Fig. 2(b). In this figure, we show three groups of samples: (a) all samples with red " $x$ " above the upper limits of XENON1T experiment [26] are ruled out, (b) those with green "o" between the upper limits of XENON1T experiment and the curve of neutrino background [47-49] are allowed and could be detectable in future detectors, and (c) the samples with blue "." below the curve of neutrino background, and they are allowed as well. However, we may not be able to distinguish the DM event from neutrino event.

\section{RELIC DENSITY AND INDIRECT SEARCH}

The matter density $(\rho)$ of the Universe is often described with a relative density $(\Omega)$

$$
\begin{gathered}
\Omega=\frac{\rho}{\rho_{\mathrm{c}}} \\
\rho_{c}=\frac{3 H_{0}^{2}}{8 \pi G_{N}} \simeq 1.88 \times 10^{-29} h^{2} \mathrm{~g} / \mathrm{cm}^{3}
\end{gathered}
$$

where $\rho_{\mathrm{c}}$ is the critical density, $G_{N}$ is Newton's gravitational constant, and $H_{0}$ is the Hubble constant, conventionally expressed as

$$
H_{0}=100 h \mathrm{~km} / \mathrm{s} / \mathrm{Mpc} \text {, }
$$

and $h \simeq 0.68$ [27].

The Planck collaboration has measured cosmological parameters with very high precision [8]. The updated cold dark matter relic density [8] is

$$
\Omega_{\mathrm{CDM}} h^{2}=0.120 \pm 0.001 .
$$

We can also take a conservative approach as demonstration that $\chi$ can be produced again in the late time by other cosmological mechanisms so that the relic density at the present at $3 \sigma$ allowed range follows

$$
\Omega_{\chi} h^{2} \leq 0.123 \text {. }
$$

This assumption also includes the standard scenario $\Omega_{\chi} h^{2} \approx 0.12$. 


\section{A. Thermal relic dark matter density}

In the early Universe, $\mathrm{DM} \chi$ existed abundantly in thermal equilibrium with other particles. The evolution of the total number density $\left(n(t)=n_{\chi}+n_{\chi^{*}}=2 n_{\chi}\right)$ for the leptonic dark matter is described by the Boltzmann equation:

$$
\frac{d n}{d t}+3 H n=-\left\langle\sigma_{\mathrm{ann}} v\right\rangle\left[n^{2}-n_{\mathrm{E}}^{2}\right],
$$

where $n_{\mathrm{E}}$ is the number density at thermal equilibrium, the Hubble parameter is

$$
H=\sqrt{4 \pi^{3} g_{*}(T) T^{4} /\left(45 M_{\mathrm{Pl}}^{2}\right)} \simeq 1.66 g_{*}^{1 / 2} T^{2} / M_{\mathrm{Pl}},
$$

$M_{\mathrm{Pl}}=1.2 \times 10^{19} \mathrm{GeV}$ is the Planck mass, $g_{*}$ is the total effective number of relativistic degrees of freedom [50,51], $\left\langle\sigma_{\mathrm{ann}} v\right\rangle$ is the thermally averaged annihilation cross section times velocity, and $v$ is the relative velocity. The relative velocity

$$
v \equiv v_{\text {lab }}=\sqrt{s\left(s-4 m_{\chi}^{2}\right)} /\left(s-2 m_{\chi}^{2}\right)
$$

and the Mandelstam variable $s=2 m_{\chi}^{2}\left(1+1 / \sqrt{1-v^{2}}\right)$ are measured in the lab frame.

The thermally averaged annihilation cross section times velocity $\left\langle\sigma_{\mathrm{ann}} v\right\rangle$ is evaluated with the Maxwell velocity distribution,

$$
\begin{aligned}
\left\langle\sigma_{\mathrm{ann}} v\right\rangle= & \frac{3 \sqrt{6}}{\sqrt{\pi} v_{0}^{3}} \int_{0}^{\infty} d v v^{2} \frac{\left(\sigma_{\mathrm{ann}} v\right)_{\chi \chi^{*}}}{2} e^{-3 v^{2} / 2 v_{0}^{2}} \\
= & \frac{x_{f}^{3 / 2}}{2 \sqrt{\pi}} \sum_{\varphi_{1}, \varphi_{2}} \int_{0}^{\infty} d v v^{2} \frac{\left[\sigma_{\mathrm{ann}}\left(\chi \chi^{*} \rightarrow \varphi_{1} \varphi_{2}\right) v\right]}{2} e^{-x v^{2} / 4} \\
& \times \theta\left[2 m_{\chi}^{2}\left(1+\frac{1}{\sqrt{1-v^{2}}}\right)-\left(m_{\varphi_{1}}+m_{\varphi_{2}}\right)^{2}\right]
\end{aligned}
$$

where $\quad x_{f} \equiv m_{\chi} / T_{f}, \quad v_{0} \equiv\left\langle v^{2}\right\rangle^{1 / 2}=\sqrt{6 / x_{f}}$ with the freeze-out temperature $T_{f}$. The second expression represents the leading contribution of DM annihilating to a pair of particles $\left(\varphi_{1}\right.$ and $\left.\varphi_{2}\right)$ in the final state. Figure 3 shows the Feynman diagrams for the dominant leptonic scalar DM annihilation processes. For each annihilation channel, we have put a step function $(\theta)$ for the threshold energy.

As the Universe cooled down, deviation of number density $\left(n_{\chi}\right)$ from thermal equilibrium would begin when the temperature reached the freeze-out temperature $\left(T_{f}\right)$. After the temperature dropped to approximately $T_{f} / 5$, the annihilation rate of the WIMP dark matter became equal to the expansion rate of universe [42], namely $n_{\chi}\left\langle\sigma_{\mathrm{ann}} v\right\rangle=H$. The relic mass density becomes

$$
\Omega_{\chi} h^{2}=n_{\chi} m_{\chi} / \rho_{\mathrm{c}}=\frac{H m_{\chi}}{\left\langle\sigma_{\mathrm{ann}} v\right\rangle \rho_{\mathrm{c}}}
$$

and the leptonic scalar $\chi$ remains as cold dark matter. From the freeze-out condition, $n_{\chi}\left\langle\sigma_{\mathrm{ann}} v\right\rangle=H$, the freeze-out temperature parameter $x_{f}$ can be solved numerically by the following equation $[42,50]$

$$
x_{f}=\ln \left[c(c+2) \sqrt{\frac{45}{8}} \frac{g_{\chi} m_{\chi} M_{\mathrm{pl}}\left(a+6 b / x_{f}\right)}{2 \pi^{3} \sqrt{g_{*}\left(m_{\chi} / x_{f}\right)} x_{f}^{1 / 2}}\right] \text {. }
$$

In the above, $c$ is an order of unity parameter determined by matching the late-time and early-time in the freeze-out criterion. The exact value of $c$ is not so significant to solve the numerical solution for $x_{f}$ due to the logarithmic dependence in Eq. (31). Following the standard procedure [50] to solve the Boltzmann equation [Eq. (26)], the relic DM density $\Omega_{\mathrm{DM}} \equiv \rho_{\chi} / \rho_{\mathrm{c}}$ can be approximately related to $\left\langle\sigma_{\mathrm{ann}} v\right\rangle$ as

$$
\Omega_{\mathrm{DM}} h^{2} \approx 1.04 \times 10^{9} \frac{\mathrm{GeV}^{-1}}{M_{\mathrm{Pl}} \sqrt{g_{*}\left(T_{f}\right)} J\left(x_{f}\right)},
$$
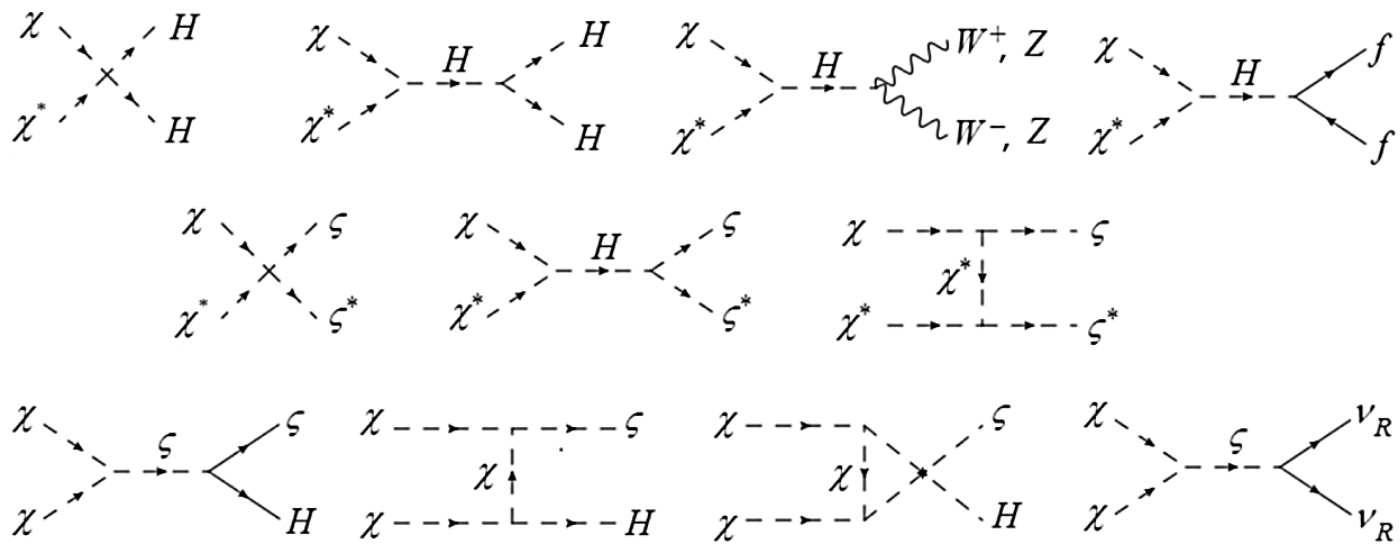

FIG. 3. Feynman diagrams for leptonic scalar DM annihilation. 
where

$$
\begin{aligned}
J\left(x_{f}\right) & \equiv \int_{x_{f}}^{\infty} \frac{\left\langle\sigma_{\mathrm{ann}} v\right\rangle}{x^{2}} d x \\
& =\int_{0}^{\infty} d v \frac{\left(\sigma_{\mathrm{ann}} v\right)_{\chi \chi^{*}}}{2} v\left[1-\operatorname{erf}\left(v \sqrt{x}_{f} / 2\right)\right] .
\end{aligned}
$$

In the nonrelativistic limit, $J\left(x_{f}\right)=a / x_{f}+3 b / x_{f}^{2}$.

The DM particles became nonrelativistic (NR) when they froze out of thermal equilibrium in the early universe. In the NR limit, we have $\sigma_{\text {ann }}\left(\chi \chi^{*} \rightarrow\right.$ all $) v=a+b v^{2}+$ $O\left(v^{4}\right)$ by applying the Taylor series expansion and its thermally averaged value Eq. (29) can be simplified as $\left\langle\sigma_{\mathrm{ann}} v\right\rangle=a+6 b / x_{f}+O\left(1 / x_{f}^{2}\right)$.

\section{B. Indirect search for leptonic scalar dark matter}

In the halo of the Milky Way and nearby galaxies, WIMP DM annihilation might generate high energy gamma-rays and appear in detectors such as Fermi-LAT [28,29], H.E.S.S. [30,52], HAWC [53], MAGIC [54], or VERITAS [55]. In addition, WIMP dark matter would lose energy when they pass through massive stars such as the sun. They become gravitationally trapped and accumulate. WIMP annihilations could be sources of high energy neutrinos and might be detected by ANTARES [56] and IceCube [57].
At present, the most stringent limits for our surveyed DM mass range, $m_{H} / 2<m_{\chi}<1000 \mathrm{GeV}$, come from FermiLAT [28,29] and H.E.S.S. [30]. We will evaluate the leptonic scalar DM annihilation cross section $\left\langle\sigma_{\text {ann }} v\right\rangle$ in different channels, and investigate the discovery potential as well as determine favored parameters guided by the Fermi-LAT and the H.E.S.S. data.

Fermi-LAT analyzed 15 dwarf spheroidal satellite galaxies (dSphs) $[28,29]$, while the H.E.S.S observed $\gamma$-ray toward the inner 300 parsecs of the Milky Way. The speed of the sun moving around the galactic center is approximately $220 \mathrm{~km} / \mathrm{s}$ at the local distance $r \approx 8.5 \mathrm{kpc}$ and the galactic circular rotation speed is about $230 \mathrm{~km} / \mathrm{s}$ at radii $\approx 100 \mathrm{kpc}$ $[42,58]$. On the other hand, the distance between the 15 $\mathrm{dSphs}$ and the sun is $\approx 23-233 \mathrm{kpc}$ [28]. In the indirectdetection calculation, we conventionally adopt a typical DM velocity $v_{0} \simeq 10^{-3}$ in the unit of the light speed [59].

In the leptonic scalar DM model, the dark matter particle $(\chi)$ can annihilate into a pair of SM particles such as $W^{+} W^{-}, Z^{0} Z^{0}, H H$, fermion pairs $f \bar{f}$, or $\zeta \zeta^{*}$ through s-channel exchange of SM Higgs boson $H$. In addition, The leptonic scalar DM can also annihilate into a pair of $\zeta \zeta^{*}$ or $H H$ through 4-point interactions and t-channel exchange of $\chi$, or $\nu_{R} \nu_{R}$ through s-channel exchange of $\zeta$, or a pair of $\zeta H$ through s-exchange of $\zeta$ and t- and $\mathrm{u}$-channel exchange of $\chi$ as presented in Fig. 3. From these Feynman diagrams, we calculate the corresponding DM annihilation cross sections at tree-level:

$$
\begin{aligned}
& (\sigma v)_{\chi \chi^{*} \rightarrow \zeta \zeta^{*}}=\frac{\sqrt{s-4 m_{\zeta}^{2}}}{16 \pi \sqrt{s}\left(s-2 m_{\chi}^{2}\right)}\left\{\lambda_{12}^{2}+\frac{16 \mu_{12}^{2}}{m_{\zeta}^{4}-m_{\chi}^{2}\left(s-4 m_{\zeta}^{2}\right)}+\frac{\lambda_{01}^{2} \lambda_{02}^{2} v^{4}-2 \lambda_{01} \lambda_{02} \lambda_{12} v^{2}\left(s-m_{H}^{2}\right)}{\left(s-m_{H}^{2}\right)^{2}+m_{H}^{2} \Gamma_{H}^{2}}\right. \\
& \left.+\frac{8 \mu_{12}^{2}\left[\lambda_{12}\left(\left(s-m H^{2}\right)^{2}+m_{H}^{2} \Gamma_{H}^{2}\right)-\lambda_{01} \lambda_{02} v^{2}\left(s-M_{H}^{2}\right)\right]}{\sqrt{\left(s-4 m_{\chi}^{2}\right)\left(s-4 m_{\zeta}^{2}\right)}\left(\left(s-m H^{2}\right)^{2}+m_{H}^{2} \Gamma_{H}^{2}\right)} \times \log \left[\frac{s-2 m_{\zeta}^{2}+\sqrt{\left(s-4 m_{\chi}^{2}\right)\left(s-4 m_{\zeta}^{2}\right)}}{s-2 m_{\zeta}^{2}-\sqrt{\left(s-4 m_{\chi}^{2}\right)\left(s-4 m_{\zeta}^{2}\right)}}\right]\right\}, \\
& (\sigma v)_{\chi \chi^{*} \rightarrow f \bar{f}}=\frac{\mathcal{C}_{f} \lambda_{01}^{2} m_{f}^{2}\left(s-4 m_{f}^{2}\right)^{3 / 2}}{8 \pi \sqrt{s}\left(s-2 m_{\chi}^{2}\right)\left[\left(s-m_{H}^{2}\right)^{2}+m_{H}^{2} \Gamma_{H}^{2}\right]}, \\
& (\sigma v)_{\chi \chi^{*} \rightarrow V V}=\frac{\lambda_{01}^{2} \sqrt{s-4 m_{V}^{2}}\left(s^{2}-4 s m_{V}^{2}+12 m_{V}^{4}\right)}{16 \pi \sqrt{s}\left(s-2 m_{\chi}\right)\left[\left(s-m_{H}^{2}\right)^{2}+m_{H}^{2} \Gamma_{H}^{2}\right]} S, \quad S= \begin{cases}1 & \text { for } W^{+} W- \\
1 / 2 & \text { for } Z^{0} Z^{0}\end{cases} \\
& (\sigma v)_{\chi \chi \rightarrow \nu_{R i} \nu_{R j}}=\frac{\mu_{12}^{2}\left|f_{i j}\right|^{2} \sqrt{s-4 m_{\nu}^{2}}\left(s-2 m_{\nu}^{2}\right)}{\pi \sqrt{s}\left(s-2 m_{\chi}^{2}\right)\left[\left(s-m_{\zeta}^{2}\right)^{2}+m_{\zeta}^{2} \Gamma_{\zeta}^{2}\right]}, \\
& (\sigma v)_{\chi \chi \rightarrow \zeta H}=\frac{\left(\lambda_{01} \mu_{12} v\right)^{2} \sqrt{A}}{2 \pi\left(s-2 m_{\chi}^{2}\right)\left(m_{\chi}^{2} A+s m_{H}^{2} m_{\zeta}^{2}\right)}+\frac{2\left(\lambda_{01} \mu_{12} v\right)^{2} s \tanh ^{-1}(\sqrt{A B} / C)}{\pi\left(s-2 m_{\chi}^{2}\right) \sqrt{B} C} \\
& -\frac{2 \lambda_{01} \lambda_{02}\left(\mu_{12} v\right)^{2}\left(s-m_{\zeta}^{2}\right) \tanh ^{-1}(\sqrt{A B} / C)}{\pi\left(s-2 m_{\chi}^{2}\right) \sqrt{B} D}+\frac{\left(\lambda_{02} \mu_{12} v\right)^{2} \sqrt{A}}{4 \pi s\left(s-2 m_{\chi}^{2}\right) D},
\end{aligned}
$$




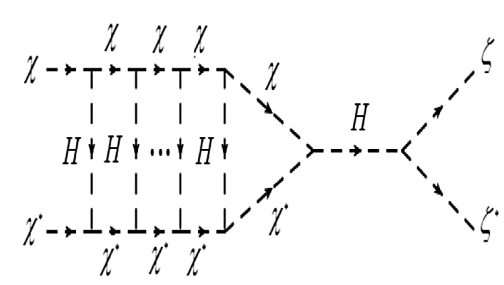

(a)

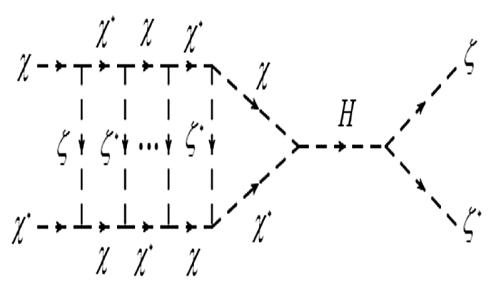

(b)

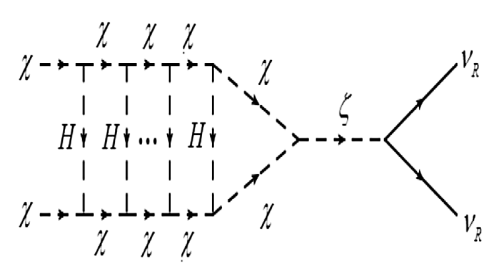

(c)

FIG. 4. Schematic diagrams for nonpertubative annihilation processes: $\chi \chi^{*} \rightarrow \zeta \zeta^{*}$ and $\chi \chi^{*} \rightarrow \nu_{R} \nu_{R}$.

where

$$
\begin{aligned}
A & =\left[s-\left(m_{H}+m_{\zeta}\right)^{2}\right]\left[s-\left(m_{H}-m_{\zeta}\right)^{2}\right], \\
B & =s\left(s-4 m_{\chi}^{2}\right), \\
C & =s\left(s-m_{H}^{2}-m_{\zeta}^{2}\right), \\
D & =\left(s-m_{\zeta}^{2}\right)^{2}+m_{\zeta}^{2} \Gamma_{\zeta}^{2} .
\end{aligned}
$$

After substituting $s=2 m_{\chi}^{2}\left(1+1 / \sqrt{1-v^{2}}\right)$ into the above equations and expanding around $v^{2}$, one can obtain the usual form: $\left\langle\sigma_{\mathrm{ann}} v\right\rangle=\left\langle a+b v^{2}+\mathcal{O}\left(v^{4}\right)\right\rangle$ in the nonrelativistic limit. As discussed in Sec. II, the decay lifetime of $\zeta$ is so short that the $\zeta$ has all decayed into two right-handed neutrinos $\nu_{R}$, and the light $\nu_{R}$ decouples from the SM particles at its freeze out temperature $T_{f}^{R} \sim m_{\zeta}$ before the onset of BBN.

\section{Sommerfeld enhancement effect}

When the DM particles froze out in the early universe, they became nonrelativistic and the nonperturbative Sommerfeld enhancement effect becomes important [60-66]. We present the schematic diagrams of annihilation processes with the Sommerfeld enhancement effect for $\chi \chi^{*} \rightarrow \zeta \zeta^{*}$ in Figs. 4(a), 4(b) and for $\chi \chi \rightarrow \nu_{R} \nu_{R}$ in Fig. 4(c). In fact, Sommerfeld effect contains an infinite series of the ladder diagrams.

Let us first consider the easier case of Sommerfeld enhancement effect on the annihilation process $\chi\left(p_{1}\right) \chi\left(p_{2}\right) \rightarrow \nu_{R}\left(p_{3}\right) \nu_{R}\left(p_{4}\right)$ through the exchange of the mediator $X=H$, as shown in Fig. 4(c). When DM particles $\chi \chi$ become non-relativistic, they rescatter off each other [Fig. 5(a)] before annihilating to $\nu_{R} \nu_{R}$. The Sommerfeld enhanced amplitude $i A_{S}$ of $\chi \chi \rightarrow \nu_{R} \nu_{R}$ annihilation process [Fig. 5(b)] can be expressed as

$$
i A_{\mathcal{S}}\left(p_{3}, p_{4}, p_{1}, p_{2}\right)=i A\left(p_{3}, p_{4}, p_{1}, p_{2}\right)+\int \frac{d^{4} p_{3}^{\prime}}{(2 \pi)^{4}} i A\left(p_{3}, p_{4} ; p_{3}^{\prime}, p_{4}^{\prime}\right)\left(i D_{F}\left(p_{3}^{\prime}\right)\right) i \Gamma\left(p_{3}^{\prime}, p_{4}^{\prime} ; p_{1}, p_{2}\right)\left(i D_{F}\left(p_{4}^{\prime}\right)\right),
$$

where $i A$ is the amplitude of the annihilation process at tree level, $D_{F}$ is the leptonic scalar DM propagator and $i \Gamma$ is the amputated nonperturbative 4-point vertex function [Fig. 5(a)] describing the rescattering process of $\chi\left(p_{1}\right) \chi\left(p_{2}\right) \rightarrow$ $\chi\left(p_{3}^{\prime}\right) \chi\left(p_{4}^{\prime}\right)$ and satisfying the following equation:

$$
i \Gamma\left(p_{3}^{\prime}, p_{4}^{\prime}, p_{1}, p_{2}\right)=i \tilde{\Gamma}\left(p_{3}^{\prime}, p_{4}^{\prime} ; p_{1}, p_{2}\right)+\int \frac{d^{4} p_{3}^{\prime \prime}}{(2 \pi)^{4}} i \tilde{\Gamma}\left(p_{3}^{\prime}, p_{4}^{\prime} ; p_{3}^{\prime \prime}, p_{4}^{\prime \prime}\right)\left[i D_{F}\left(p_{3}^{\prime \prime}\right)\right] i \Gamma\left(p_{1}, p_{2} ; p_{3}^{\prime \prime}, p_{4}^{\prime \prime}\right)\left[i D_{F}\left(p_{4}^{\prime \prime}\right)\right] .
$$

In the above, we have the lowest order perturbative 4-point vertex function given by

$$
i \tilde{\Gamma}\left(p_{3}^{\prime}, p_{4}^{\prime} ; p_{3}^{\prime \prime}, p_{4}^{\prime \prime}\right)=-i g_{X}^{2} \frac{1}{\left(p_{3}^{\prime \prime}-p_{3}^{\prime}\right)^{2}-m_{X}^{2}},
$$

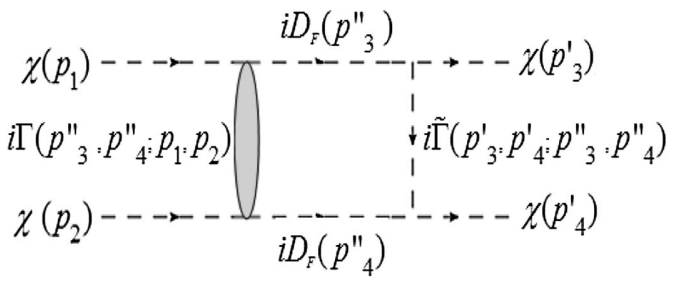

(a)

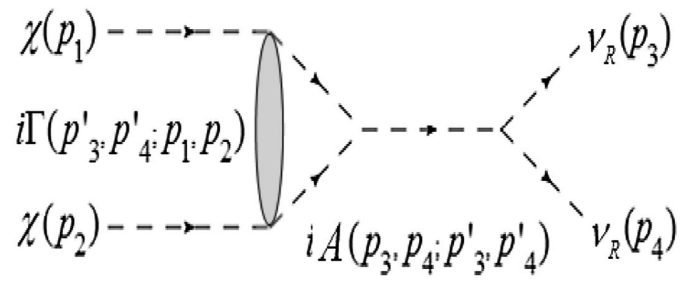

(b)

FIG. 5. Diagrams for (a) rescattering of $\chi \chi \rightarrow \chi \chi$, and (b) nonperturbative annihilation of $\chi \chi \rightarrow \nu_{R} \nu_{R}$. 
where $X$ is the mediator particle $(X=H)$, and $p_{4}^{\prime \prime}=-p_{3}^{\prime \prime}+p_{3}^{\prime}+p_{4}^{\prime}$. Note that the dimensionless coupling strength of DM $\chi$ with the mediator $X$ is $g_{X}=g_{X}^{\prime} /\left(2 m_{\chi}\right)$, where the dimensionful coupling $g_{X}^{\prime}$ is usually defined in the interaction Lagrangian. When the process is mediated by the Higgs boson $(H)$, we have $g_{H}=g_{H}^{\prime} /\left(2 m_{\chi}\right)=\lambda_{01} \mathrm{v} /\left(2 m_{\chi}\right)$, where the dimensionful coupling $g_{H}^{\prime}=\lambda_{01} \mathrm{v}$ as described in the scalar potential [Eq. (1)].

From Appendix A 1, we find that the pair of DM particles $\chi \chi$ form a bound state and the wave function $\psi(\vec{r})$ satisfies the following Schrödinger equation:

$$
-\frac{1}{2 \mu} \nabla^{2} \psi(\vec{r})+V(\vec{r}) \psi(\vec{r})=E \psi(\vec{r})=\frac{1}{2} \mu v^{2} \psi(\vec{r}) .
$$

where $\mu=m_{\chi} / 2$ is the reduce mass of the bound state $(\chi \chi)$, and $E=|\vec{p}|^{2} / 2 \mu \equiv \mu v^{2} / 2$ is the total kinetic energy with the relative velocity $v=v_{\text {lab }}$ defined in Eq. (28). $V(r)$ is a Yukawa-type potential

$$
V(r)=-\alpha_{X} \frac{e^{-m_{X} r}}{r}
$$

where $\alpha_{X}=g_{X}^{2} / 4 \pi$ and $m_{X}$ is the mass of mediator.

For the case of s-wave rescattering, the Sommerfeld enhanced amplitude $i A_{\mathcal{S}}$ can be written as $i A_{\mathcal{S}}\left(p_{1}, p_{2} ; p_{3}, p_{4}\right)=i A\left(\vec{p}_{1}, \vec{p}_{2} ; \vec{p}_{3}, \vec{p}_{4}\right) \psi(\vec{r}=0)$,

where $i A$ is the amplitude at tree level as explained in Appendix A 2. Consequently, the Sommerfeld enhanced velocity averaged annihilation cross section $\langle\sigma v\rangle_{\mathcal{S}} \simeq a_{\mathcal{S}}+$ $b_{\mathcal{S}} v^{2}$ can be further simplified as

$\langle\sigma v\rangle_{\mathcal{S}} \sim a_{\mathcal{S}}=\langle a \mathcal{S}(v)\rangle, \quad \mathcal{S}=\left|\psi_{l=0}(\vec{r}=0)\right|^{2}$,

where $\mathcal{S}$ is just the s-wave Sommerfeld enhancement factor (see Appendix A 2).

It is well known that there is no analytical solution with a Yukawa potential in Eq. (43), but the Hulthén potential maintains the same short and long distance behavior of the Yukawa potential and has an analytical solution for s-wave function. Hence it is a good approximation to employ the Hulthén potential to obtain $\left|\psi_{l=0}(\vec{r}=0)\right|$ with a Yukawa potential [67],

$$
V(\vec{r}) \simeq-\alpha_{X} \frac{\left(\pi^{2} m_{X} / 6\right) e^{-\pi^{2} m_{X} r / 6}}{1-e^{-\pi^{2} m_{X} r / 6}},
$$

where the mediator particle $X=H$. One can obtain the wave function [68] in terms of Gamma function $\Gamma$

$\psi_{l=0}(\vec{r}=0)=i \frac{\pi^{2} \epsilon_{X} / 6}{2 \epsilon_{v}} \Gamma\left(1-i \frac{\epsilon_{v}}{\pi^{2} \epsilon_{X} / 6}\left(1+\sqrt{1-\frac{\pi^{2} \epsilon_{X} / 6}{\epsilon_{v}^{2}}}\right)\right) \times \Gamma\left(1-i \frac{\epsilon_{v}}{\pi^{2} \epsilon_{X} / 6}\left(1-\sqrt{1-\frac{\pi^{2} \epsilon_{X} / 6}{\epsilon_{v}^{2}}}\right)\right) / \Gamma\left(\frac{-2 i \epsilon_{v}}{\pi^{2} \epsilon_{X} / 6}\right)$,

with $^{1}$

$$
\epsilon_{v} \equiv \frac{v}{2 \alpha_{X}}, \quad \epsilon_{X} \equiv \frac{m_{X}}{\alpha_{X} m_{\chi}},
$$

and the s-wave Sommerfeld factor is given by [69]

$$
\begin{aligned}
\mathcal{S} & \left(m_{\chi}, m_{X}, \alpha_{X}, v\right) \\
& =\left|\psi_{l=0}(\vec{r}=0)\right|^{2} \\
& =\frac{\pi}{\epsilon_{v}} \frac{\sinh \left(\frac{2 \pi \epsilon_{v}}{\pi^{2} \epsilon_{X} / 6}\right)}{\cosh \left(\frac{2 \pi \epsilon_{v}}{\pi^{2} \epsilon_{X} / 6}\right)-\cos \left(2 \pi \sqrt{\frac{1}{\pi^{2} \epsilon_{X} / 6}-\frac{\epsilon_{v}^{2}}{\left(\pi^{2} \epsilon_{X} / 6\right)^{2}}}\right)} .
\end{aligned}
$$

For $\chi \chi \rightarrow \nu_{R} \nu_{R}$ annihilation process, the mediator particle is $X=H . \psi_{l=0}(\vec{r}=0)$ indeed goes to 1 in the $\alpha_{X}=0$ limit. We will see that the analytic solution $\mathcal{S}=\left|\psi_{l=0}(\vec{r}=0)\right|^{2}$

\footnotetext{
${ }^{1}$ Note that the $\beta$ in the formula of [67] is in fact $v / 2$ in this work.
}

agrees well with that obtained from numerically solving the Schrödinger equation with the Yukawa potential.

When the mediator mass can be neglected, the Yukawa potential can be approximated by a Coulomb potential:

$$
V(r) \simeq-\frac{\alpha_{X}}{r}
$$

The corresponding s-wave function is given by [70]

$\psi_{l=0}^{(\mathrm{coul})}(\vec{r})=\Gamma(1+i \gamma) e^{-\pi \gamma / 2} e^{i \vec{p} \cdot \vec{r}}{ }_{1} \mathcal{F}_{1}(-i \gamma, 1, i p r-i \vec{p} \cdot \vec{r})$,

where ${ }_{1} \mathcal{F}_{1}$ is the confluent hypergeometric function of the first kind, and

$$
\gamma=\frac{\alpha_{X}}{v}=\frac{\alpha_{X} \mu}{|\vec{p}|}
$$

In this approximation we have 


$$
\psi_{l=0}^{(\mathrm{coul})}(\vec{r}=0)=\Gamma(1+i \gamma) e^{-\pi \gamma / 2} .
$$

Accordingly, the corresponding s-wave Sommerfeld factor in the Coulomb potential is

$$
\begin{aligned}
\mathcal{S}^{(\mathrm{coul})} & =\left|\psi_{l=0}^{(\mathrm{coul})}(\vec{r}=0)\right|^{2} \\
& =\Gamma(1+i \gamma) \Gamma(1-i \gamma) e^{-\pi \gamma}=\frac{2 \pi \gamma}{e^{2 \pi \gamma}-1} .
\end{aligned}
$$

In fact $\left|\psi_{l=0}(\vec{r}=0)\right|$ goes to $\left|\psi_{l=0}^{\operatorname{coul}}(\vec{r}=0)\right|$ and the Sommerfeld factor $\mathcal{S}$ in Eq. (50) does reduce to $\mathcal{S}^{\text {(coul) }}$ in the large $m_{\chi}$ region [67,71].

As mentioned above, we only keep the first term (the $a$ term) in Eqs. (34)-(35) for both relic density calculation and the indirect annihilation processes, namely, the s-wave contribution, with $\left\langle\sigma_{\mathrm{ann}} v\right\rangle \approx a+\mathcal{O}\left(v^{2}\right)$. Hence we show the $a$-term for each annihilation process as follows:

$$
\begin{aligned}
a^{\zeta \zeta^{*}}= & \frac{\sqrt{m_{\chi}^{2}-m_{\zeta}^{2}}}{32 \pi m_{\chi}^{3}}\left\{\frac{\left(\mu_{12}^{2}-\lambda_{12} m_{\zeta}^{2}+2 \lambda_{12} m_{\chi}^{2}\right)^{2}}{\left(2 m_{\chi}^{2}-m_{\zeta}^{2}\right)^{2}}\right. \\
& +\frac{\lambda_{01}^{2} \lambda_{02}^{2} v^{4}}{\left[\left(4 m_{\chi}^{2}-m_{H}^{2}\right)^{2}+m_{H}^{2} \Gamma_{H}^{2}\right]} \\
& \left.-\frac{2 \lambda_{01} \lambda_{02} v^{2}\left(4 m_{\chi}^{2}-m_{H}^{2}\right)\left(4 \mu_{12}^{2}-\lambda_{12} m_{\zeta}^{2}+2 \lambda_{12} m_{\chi}^{2}\right)}{\left(2 m_{\chi}^{2}-m_{\zeta}^{2}\right)\left[\left(4 m_{\chi}^{2}-m_{H}^{2}\right)^{2}+m_{H}^{2} \Gamma_{H}^{2}\right]}\right\},
\end{aligned}
$$

$$
\begin{gathered}
a^{f \bar{f}}=\frac{C_{f} \lambda_{01}^{2} m_{f}^{2}\left(m_{\chi}^{2}-m_{f}^{2}\right)^{3 / 2}}{4 \pi m_{\chi}^{3}\left[\left(4 m_{\chi}^{2}-m_{H}^{2}\right)^{2}+m_{H}^{2} \Gamma_{H}^{2}\right]}, \\
a^{V V}=\frac{\lambda_{01}^{2} \sqrt{m_{\chi}^{2}-m_{W}^{2}}\left(4 m \chi^{4}-4 m_{\chi}^{2} m_{W}^{2}+3 m_{W}^{4}\right)}{\left.8 \pi m_{\chi}^{3}\left(\left(4 m_{\chi}^{2}-m_{H}^{2}\right)^{2}+m_{H}^{2} \Gamma_{H}^{2}\right)\right)} S, \\
a^{\zeta H}=\frac{\mu_{12}^{2} v^{2}}{128 \pi m_{\chi}^{4} D}\left\{4 \sqrt{A}\left[\lambda_{02}^{2}+\frac{8 \lambda_{01}^{2} D\left(A+4 m_{H}^{2} m_{\zeta}^{2}\right)}{\left(A+4 m_{H}^{2} m_{\zeta}^{2}\right)^{2}}\right]\right. \\
\left.-\frac{128 \lambda_{01} m_{\chi}^{2}\left[\lambda_{02}\left(4 m_{\chi}^{2}-m_{\zeta}^{2}\right) C-8 \lambda_{01} m_{\chi}^{2} D\right]}{\sqrt{B} C}\right\} .
\end{gathered}
$$

For $\chi \chi \rightarrow \nu_{R} \nu_{R}$ and $\zeta H$ annihilation processes, we have

$$
\begin{aligned}
\langle\sigma v\rangle_{\mathcal{S}}\left(\chi \chi \rightarrow \nu_{R} \nu_{R}\right) & \simeq\left\langle a^{\nu_{R} \nu_{R}} \mathcal{S}\left(m_{\chi}, m_{H}, \alpha_{H}, v\right)\right\rangle, \\
\langle\sigma v\rangle_{\mathcal{S}}(\chi \chi \rightarrow \zeta H) & \simeq\left\langle a^{\zeta H} \mathcal{S}\left(m_{\chi}, m_{H}, \alpha_{H}, v\right)\right\rangle .
\end{aligned}
$$

Accordingly, we can analytically obtain the Sommerfeld factor $\mathcal{S}=\left|\psi_{l=0}(\vec{r}=0)\right|^{2}$ for the $\chi \chi \rightarrow \nu_{R} \nu_{R}$ and $\chi \chi \rightarrow \zeta H$ annihilation processes.

Figure 6 shows the s-wave Sommerfeld factor in the present universe with $\left(v=10^{-3} c\right)$ as a function of the DM mass $\left(m_{\chi}\right)$ for two values of the coupling $g_{X}^{\prime} / \mathrm{v}=10[(\mathrm{a})$ and (b)] as well as $g_{X}^{\prime} / \mathrm{v}=1$ [(c) and (d)]. The left panels [(a) and (c)] present the analytical results with the Hulthén potential [Eq. (47)] and $m_{X}=m_{H}$, as well as numerical results with Yukawa potential [Eq. (44)] and $m_{X}=m_{H}$. In addition, we show the analytical solution in Coulomb potential approximation. In the right panels [(b) and (d)], the analytical solutions in Huthén potential approximation are presented with three values of the mediator mass $m_{X}=0.01,0.1$, and $1 \mathrm{GeV}$. The Coulomb potential approximation in the left panels and the analytical solution with $m_{X}=0.01 \mathrm{GeV}$ in the right panels are both represented with orange dot-dashed lines because there is no difference between them numerically.

We find that all curves in Figs. 6(b) and 6(d) with a massive $m_{X}$ oscillate with an amplitude that increases with an increasing mediator mass, while the frequency of oscillation increases with a decreasing mediator mass. Comparing Figs. 6(a)-6(d), we see that the Sommerfeld enhancement factor $\mathcal{S}$ decreases with a decreasing coupling $g_{X}^{\prime}$. Furthermore, the strength of Sommerfeld enhancement decreases with an increasing $m_{\chi}$. The reason can be seen as follows:

(i) First, these curves oscillate around the curve generated by the Coulomb potential.

(ii) The Coulomb potential approximation provides the central value for $\mathcal{S}$.

(iii) From Eqs. (53) and (55), we note that $\mathcal{S}^{(\text {coul) }}$ is a function of $\alpha_{X}=g_{X}^{2} / 4 \pi$. Hence the enhancement factor $\mathcal{S}$ is suppressed by an increasing $m_{\chi}$ owing to $g_{X}=g_{X}^{\prime} /\left(2 m_{\chi}\right)$.

In Fig. 7, we show $\mathcal{S}$ at the early universe with $(v=0.5 \times c)$ as a function of $m_{\chi}$. The colored scheme is the same as Fig. 6 while the thick and thin lines are based on $g_{X}^{\prime} / \mathrm{v}=10$ and $g_{X}^{\prime} / \mathrm{v}=1$, respectively. Clearly, comparing with low-velocity DM in present universe as shown in Fig. 6, the nonperturbative effect for highvelocity becomes much weaker resulting in much smaller Sommerfeld enhancement in the early universe.

From Figs. 6 and 7, we find that the larger coupling strength or smaller DM relative velocity $v$ gives a greater value of the Sommerfeld factor. Roughly speaking, the Sommerfeld factor used at the present stage $\left(v \simeq 10^{-3} c\right)$ is $2 \sim 3$ order of magnitude greater than that in freeze-out stage $(v \simeq 0.5 \times c)$ with a light mediator mass. This main characteristics of velocity dependent SICS is used to solve the small scale problem.

We have shown in Figs. 6 and 7 that the numerical result agrees well with the analytic solution in Hulthén approximation at $m_{X}=m_{H}$. Nevertheless, we see that it is 


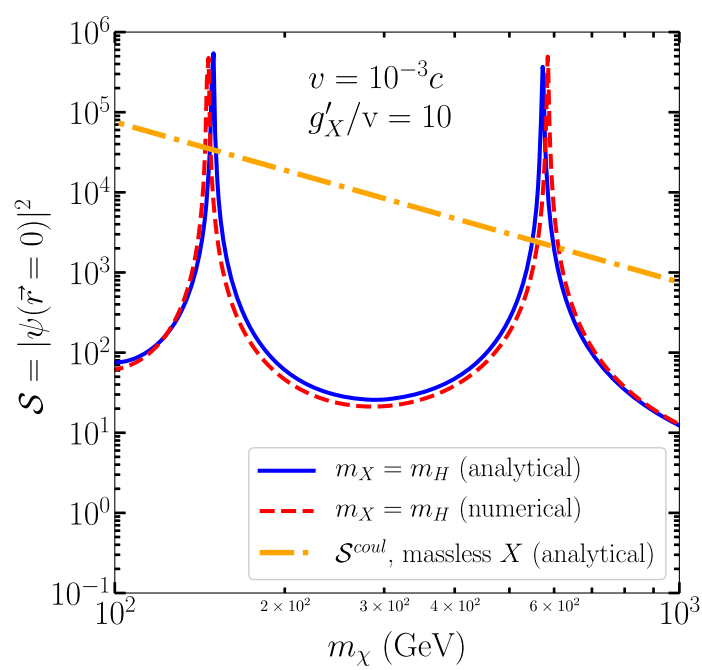

(a) Analytical and numerical solutions.

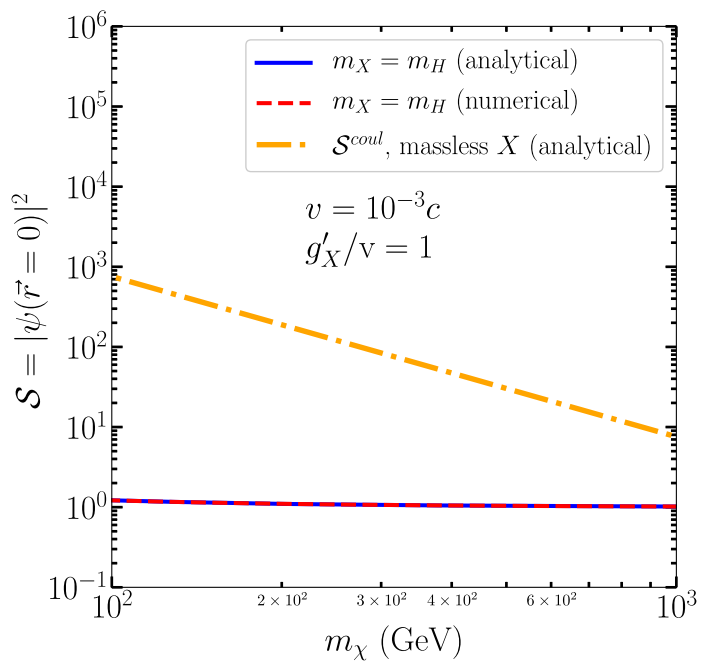

(c) Analytical and numerical solutions.

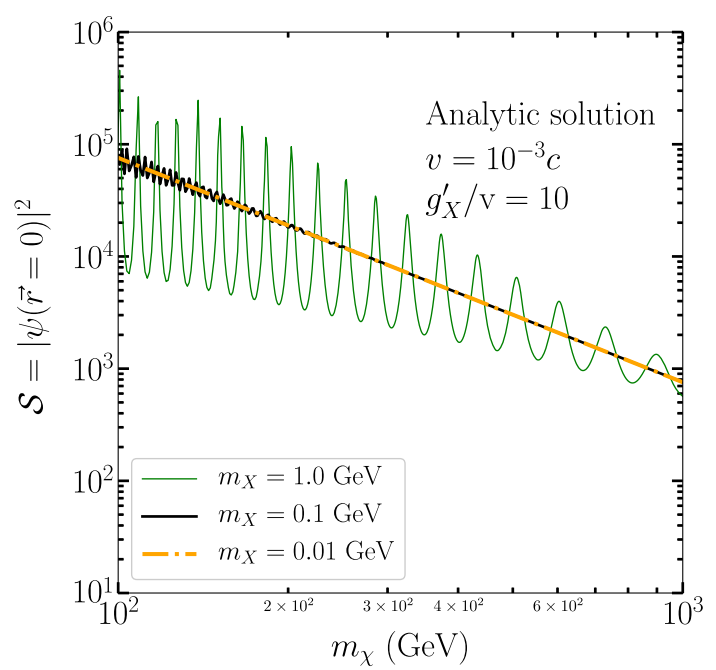

(b) Three different benchmark masses $m_{X}$.

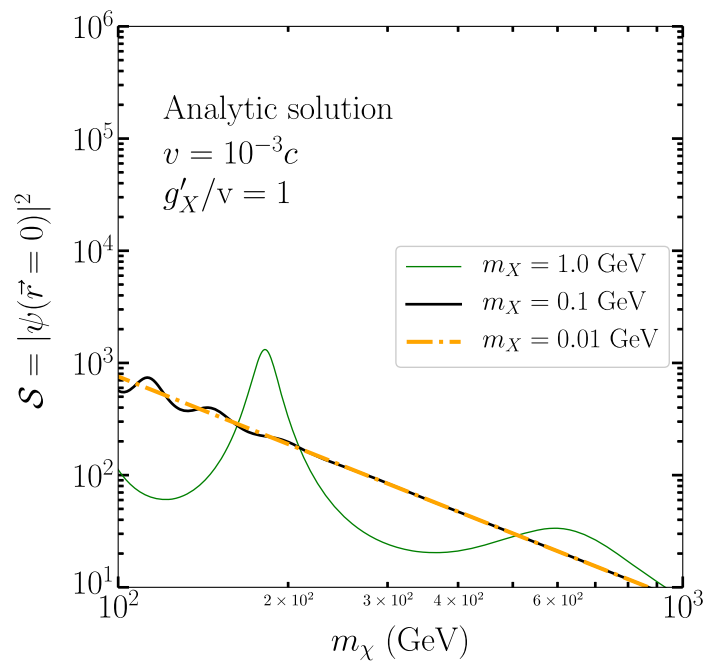

(d) Three different benchmark masses $m_{X}$.

FIG. 6. Sommerfeld factor $\mathcal{S}$ versus $m_{\chi}$ for the present universe with $v=10^{-3} c$. The $g_{X}^{\prime}$ parameter is the dimensionful coupling between the DM $(\chi)$ and the mediator $X$. The left panels [(a) and (c)] present the analytical results (blue solid) with the Hulthén potential [Eq. (47)] and $m_{X}=m_{H}$, as well as numerical results (red dashed) with Yukawa potential [Eq. (44)] and $m_{X}=m_{H}$. For comparison, the analytical solution in Coulomb potential approximation is depicted by orange dashed lines. In the right panels [(b) and (d)], the analytical solutions in Huthén potential approximation are shown with three values of the mediator mass $m_{X}=0.01$ (orange dot-dashed), 0.1 (black solid), and $1 \mathrm{GeV}$ (green solid).

impossible to obtain an analytical form for $\mathcal{S}$ in the process of $\chi \chi^{*} \rightarrow \chi^{*} \chi$, which simultaneously involves the exchanges of $H$ and $\zeta$ particles schematically shown in Figs. 4(a) and 4(b). The potential then becomes the sum of two Yukawa-type potential

$$
V(r)=-\alpha_{H} \frac{e^{-m_{H} r}}{r}-\alpha_{\zeta} \frac{e^{-m_{\zeta} r}}{r} .
$$

where $\alpha_{X}=g_{X}^{2} / 4 \pi,(X=H, \zeta), g_{H}=\lambda_{01} \mathrm{v} / 2 m_{\chi}$, and $g_{\zeta}=$ $\mu_{12} / m_{\chi}$. There is no analytical form for the Sommerfeld enhancement factor $\mathcal{S}$. Therefore, we develop a numerical solution for $\mathcal{S}$ as given in Appendix A 3 .

\section{Numerical results for relic density and indirect search}

In this subsection, we present our numerical results for the indirect search and the relic density. For the indirect search, we compare our theoretical results with the most stringent limits from the Fermi-LAT [28,29] and the H.E.S.S. results [30]. Both Fermi-LAT and H.E.S.S. astrophysical observations do not show the significant $\gamma$-ray 


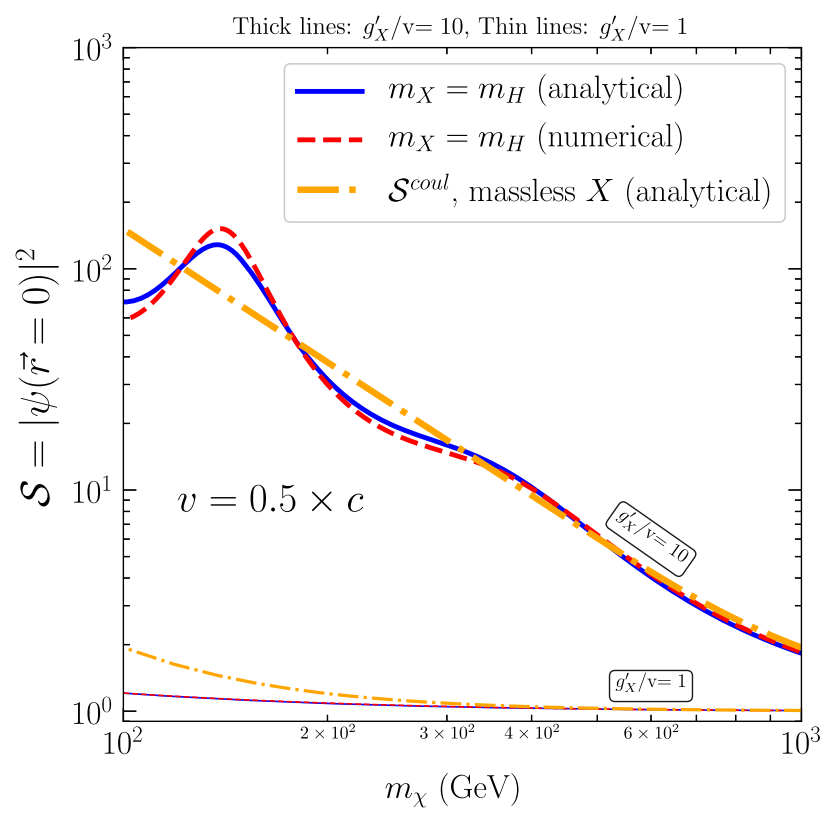

FIG. 7. Sommerfeld factor $\mathcal{S}$ versus $m_{\chi}$ for the early universe with $v=0.5 \times c$. The color scheme is same as Fig. 6. The coupling $g_{X}^{\prime} / \mathrm{v}$ for the upper thick lines are fixed to 10 while the value of the bottom thin lines are 1 .

signal above background. Instead, Fermi-LAT provides upper limits on $\left\langle\sigma_{\mathrm{ann}} v\right\rangle$ for DM annihilating into $W^{+} W^{-}$ and the SM fermion pairs: $b \bar{b}, u \bar{u}, \tau^{+} \tau^{-}, \mu^{+} \mu^{-}, e^{+} e^{-}$at 95\% confidence level with WIMPs masses between $2 \mathrm{GeV}$ to $10 \mathrm{TeV}$, while H.E.S.S. gives the upper limits on $\left\langle\sigma_{\mathrm{ann}} v\right\rangle$ for DM annihilating into $W^{+} W^{-}$and the SM fermion pairs: $t \bar{t}, b \bar{b}, \tau^{+} \tau^{-}, \mu^{+} \mu^{-}$with masses from $160 \mathrm{GeV}$ to $70 \mathrm{TeV}$.

Figure 8 presents $\left\langle\sigma_{\mathrm{ann}} v\right\rangle$ for leptonic scalar DM $(\chi \chi)$ annihilating into $W^{+} W^{-}, t \bar{t}, \tau^{+} \tau^{-}, \zeta H$ and $\zeta \zeta^{*}$. The samples above the upper limits of Fermi-LAT and H.E.S.S are ruled out. The plots on the left-handed do not include the Sommerfeld effect but the Sommerfeld effect are considered in the plots on the right-handed side. In each plot on the left-handed side, the same color scheme as presented in Fig. 2 is used. In the panels of the right column, we show the Sommerfeld effect for the data survived from XENON1T limits, namely the samples taken from those green (circle) and blue (dot) in the left panels.

Without considering the Sommerfeld effect, we see that DM can only be detected with $m_{\chi} \gtrsim 1 \mathrm{TeV}$ via $W^{+} W^{-}$or the Higgs resonance annihilation via $b \bar{b}$ and $\tau^{+} \tau^{-}$channel. Clearly, the cross sections can be enhanced by the Sommerfeld effect. Albeit the enhancement differs from channel to channel, it is interesting that the cross section of the channel $\zeta \zeta^{*}$ is overall enhanced. We see that DM annihilating to a pair of $\zeta \zeta^{*}$ is dominant while this channel is not detectable because $\zeta$ eventually decays to $\nu_{R}$.

Figure 9 shows the leptonic scalar DM thermal relic density $\Omega_{\chi} h^{2}$ as a function of $m_{\chi}$ : (a) without Sommerfeld enhancement, and (b) with Sommerfeld enhancement effects. We maintain the same symbols for each sample used in Figs. 2 and 8. The horizontal line denotes the observed relic density: $\Omega_{\text {obs }} h^{2}=0.120$ [27]. Since the relic density is roughly proportional to the inverse of the total $\left\langle\sigma_{\mathrm{ann}} v\right\rangle$, the samples are oriented reversely in vertical direction. With Sommerfeld enhancement effect, the same parameters will lead to a smaller relic density as expected. Thus, there are more regions of the parameter space satisfying the relic density requirement $\Omega_{\chi} h^{2}<0.123$ [27].

\section{COSMOLOGICAL CONSTRAINTS ON THE RIGHT-HANDED NEUTRINO}

The left-handed neutrino decouple at the temperature $T_{f}^{L} \sim 1 \mathrm{MeV}$ when the left-handed neutrinos and the righthanded antineutrinos cannot be converted to pairs of electron and positron. On the other hand, the right-handed neutrinos decouple at the temperature $T_{f}^{R} \sim m_{\zeta}$ when the production of the $\zeta$ particles is kinetically not allowed. At the temperature $T<T_{f}^{L}<T_{f}^{R}$, the total density of radiation $\rho_{r}$ is

$$
\rho_{r}=\rho_{\gamma}+\rho_{L}+\rho_{R}=3\left[1+N_{\text {eff }} \frac{7}{8}\left(\frac{4}{11}\right)^{4 / 3}\right] \rho_{\gamma},
$$

where $\rho_{\gamma}, \rho_{L}$ and $\rho_{R}$ are the energy density of photons, $\nu_{L}$, and $\nu_{R}$, respectively. The relativistic degree of freedom $N_{\text {eff }}$ here depends on the relativistic particle species and their internal degree of freedoms. Considering only three generations of left-handed neutrinos in the SM, the theoretical prediction is given by $N_{\text {eff }}=3.045[72,73]$. The recent Planck 2018 data shows $N_{\text {eff }}=2.92_{-0.37}^{+0.36}(95 \% \mathrm{CL})$ and this is compatible with the SM prediction.

Following the computation in Ref. [74], the additional contribution to the relativistic degree of freedom arising from $\nu_{R}$ is given by

$$
\Delta N_{\text {eff }}=3 \times\left[\frac{g_{* s}\left(T_{f}^{L}\right)}{g_{* s}\left(T_{f}^{R}\right)}\right]^{4 / 3} .
$$

Here, factor 3 describes three generations of neutrinos. As shown in Ref. [74], the new relativistic degree of freedom is limited to be $\Delta N_{\text {eff }}=0.10_{-0.43}^{+0.44}$.

We would like to note that both $\nu_{L}$ and $\nu_{R}$ completely decouple from the SM plasma before big bang nucleosynthesis (BBN) in this model, thus one can obtain $g_{* s}\left(T_{f}^{L}\right)=10.75$ at $T_{f}^{L} \sim 1 \mathrm{MeV}$ and $g_{* s}\left(T_{f}^{R}\right)=67$ at $0.2 \mathrm{GeV} \lesssim T_{f}^{R} \lesssim 1.2 \mathrm{GeV}$. By plugging these two values into Eq. (65), we can simply verify that $\Delta N_{\text {eff }}=0.26$ also agrees with current limit [27]. Moreover, it has also pointed out in Ref. [74] that a combined constraint from Planck CMB data and BBN (the helium abundance measurements) reads $\Delta N_{\text {eff }} \lesssim 0.53$ at $95 \%$ C.L. which associates $T_{f}^{R} \gtrsim 200 \mathrm{MeV}$. This implies $m_{\zeta} \gtrsim 200 \mathrm{MeV}$ 

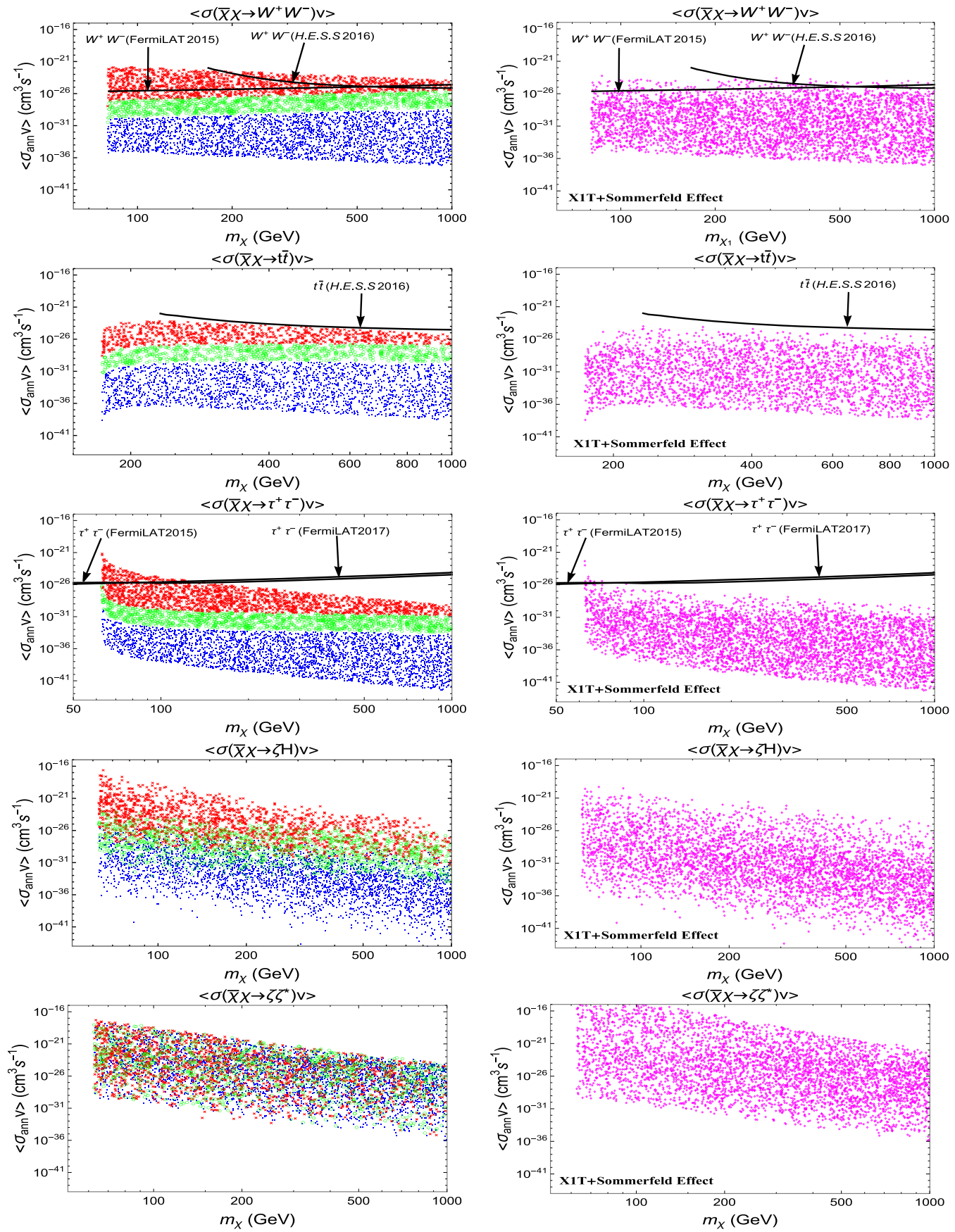

FIG. 8. Thermally averaged annihilation cross section times velocity for the leptonic scalar dark matter $(\chi)\left\langle\sigma_{\mathrm{ann}} v\right\rangle$ in different channels. Left: without the Sommerfeld effect: red (cross), green (circle) and blue (dot) denote the scenario excluded by XENON1T, testable soon by future underground detectors, and below the neutrino floor, respectively. Right: the Sommerfeld effect for those samples surviving from XENON1T limit, namely green (circle) and blue (dot) in the left panels.

if taking $T_{f}^{R} \simeq m_{\zeta}$. Therefore, to escape the combined constraint from $C M B$ and $B B N$, we always take a safe limit $m_{\zeta} \gtrsim 200 \mathrm{MeV}$ in this work.

\section{SMALL SCALE PROBLEM}

As aforementioned in the introduction, the momentum transfer cross section $\sigma_{T}$ of the process $\chi \chi^{*} \rightarrow \chi^{*} \chi$ depends on the root mean square velocity $v_{0}$ of the DM particles. To solve the small scale (CCP/MSP/TBTF) problems, we simplify to use the following constraint $[16,17]$ :

$$
0.1 \mathrm{~cm}^{2} / \mathrm{g} \leq\left\langle\sigma_{T} / m_{\chi}\right\rangle_{S} \leq 10 \mathrm{~cm}^{2} / \mathrm{g}
$$

where $\left\langle\sigma_{T} / m_{\chi}\right\rangle_{S}$ is the Sommerfeld-enhanced cross section per unit DM mass. 


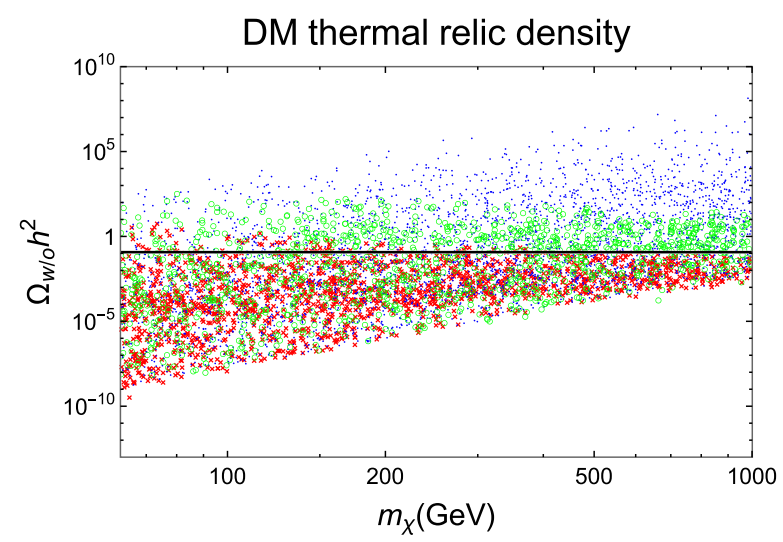

(a) Sommerfeld effect not included

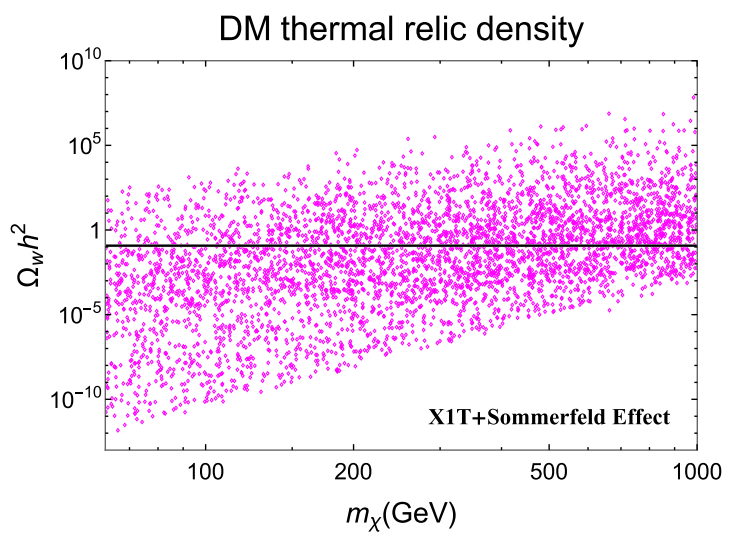

(b) Sommerfeld effect included

FIG. 9. Relic density of leptonic scalar DM $\Omega_{\chi} h^{2}$ versus $m_{\chi}$ : enhancement.

The process of $\chi \chi^{*} \rightarrow \chi^{*} \chi$ can occur via the exchanges of $H$ or $\zeta$ in the $s$ - as well as $t$-channels. It can also occur via the $\lambda_{1}$ quartic term interaction. The subleading terms via the $s$-channel for the Sommerfeld effect can be ignored [75]. For the $t$-channel contribution, in the NR limit, we have

$$
\sigma_{T}=\frac{\left(4 \mu_{12}^{2} m_{H}^{2}+\lambda_{01}^{2} \mathrm{v}^{2} m_{\zeta}^{2}\right)^{2}}{64 \pi m_{\zeta}^{4} m_{H}^{4} m_{\chi}^{2}} \simeq \frac{\mu_{12}^{4}}{4 \pi m_{\zeta}^{4} m_{\chi}^{2}} .
$$

The approximation holds only if $\mu_{12} \gg \lambda_{01} \mathrm{v} m_{\zeta} /\left(2 m_{H}\right)$. We find that this approximation can be applied for the majority of our collected samplings. Note that we have ignored the quartic interaction since $\mu_{12} / m_{\zeta} \gg \lambda_{01} \mathrm{v} /\left(2 m_{H}\right)>\sqrt{\lambda_{1} / 8}$. For example, we can have $\lambda_{01} \mathrm{v} /\left(2 m_{H}\right)=1.85$ and $\sqrt{\lambda_{1} / 8}=0.67$ by taking $\lambda_{01}=\lambda_{1}=\sqrt{4 \pi}$.

\section{MODEL PARAMETER SPACE}

In the $C P$-conserving LSDM model, there are eight free real parameters:

$$
m_{\chi}, m_{\zeta}, \mu_{12}, \lambda_{1}, \lambda_{2}, \lambda_{01}, \lambda_{02}, \quad \text { and } \lambda_{12}
$$

In this section, we look for favored regions of the parameter space in the LSDM model with implications from astrophysical and cosmological observations. We apply selection requirements for small scale structure, cold dark matter relic density, direct searches, and indirect detections, as well as cosmological constraints on right handed neutrinos.

For the leptonic scalar DM, the solution of small scale problem comes from DM strong self-interaction with $\sigma_{T} / m_{\chi}$ that contains 4 free parameters: $m_{\chi}, m_{\zeta}, \mu_{12}$, and $\lambda_{01}$, as shown in Eq. (67). At first, we perform a grid scan for the ranges, $m_{H} / 2<m_{\chi}<1200 \mathrm{GeV}$, $0.2<m_{\zeta} / \mathrm{GeV}<1$, and $-6<\log _{10}\left[\lambda_{01}\right]<\log _{10}[\sqrt{4 \pi}]$. With these collected samples, we can pin down the corresponding allowed range for $\mu_{12}$ by using the allowed range of $\sigma_{T} / m_{\chi}$. Second, we use the interpolation technique to find the allowed range of $\mu_{12}$ from random sampling for $m_{\chi}, m_{\zeta}$ and $\lambda_{12}$. We then apply random sampling with the 8 free parameters as usual except that $\mu_{12}$ is chosen from the allowed region with randomly selected $m_{\chi}, m_{\zeta}$ and $\lambda_{12}$. Third, we use the selected parameters to find the allowed samples which satisfy the observed relic density constraint. Finally, we find the allowed parameter space by satisfying the constraints from the direct and indirect searches.

Following this procedure, we collect a thousand samples that satisfy all the constraints mentioned above. Figure 10 shows the predictions in the LSDM model for $\sigma_{T} / m_{\chi}$, $\Omega_{\chi} h^{2}, \sigma^{S I}$, and $\left\langle\sigma_{\text {ann }} v\right\rangle$ in $W^{+} W^{-}, t \bar{t}, \tau^{+} \tau^{-}, \zeta H$ and $\zeta \zeta^{*}$ channels. Comparing the scenarios with and without Sommerfeld effect, we only depict those samples in agreement with XENON1T data in Fig. 10. The Sommerfeld effect is applied in the computation for the magenta samples (diamond), while Green (circle) (testable for near future) and blue (filled circle) (below the neutrino floor) are obtained without including the Sommerfeld effect. It is clear to see in Fig. 10(a), that the $\left\langle\sigma_{T} / m_{\chi}\right\rangle_{S}$ is enhanced by the Sommerfeld effect such that all values fall into $0.1\left(\mathrm{~cm}^{2} / \mathrm{g}\right) \leq\left\langle\sigma_{T} / m_{\chi}\right\rangle_{S} \leq 10\left(\mathrm{~cm}^{2} / \mathrm{g}\right)$. In Fig. 10(b), we see that the relic density is roughly proportional to $1 /\left\langle\sigma_{\text {ann }}\right\rangle$, and hence the $\Omega_{\chi} h^{2}$ becomes suppressed by the Sommerfeld effect such that the relic density of all selected samples fall into the range of $\Omega_{\mathrm{obs}} h^{2} \pm 3 \sigma$.

It is interesting that the selected 1000 samples satisfying the small scale requirement (CCP/MSP/TBTF) and the observed relic density constraints also satisfy the constraint on the SI DM-nucleon scattering cross section $\sigma_{\chi p}^{S I}$ shown in Fig. 10(c). We have extended XENON1T 2018 data to $m_{\chi} \simeq 1.2 \mathrm{TeV}$ with the dashed line. The cross section $\left\langle\sigma_{\text {ann }} v\right\rangle$ for DM annihilating into $W^{+} W^{-}, t \bar{t}, \tau^{+} \tau^{-}, \zeta H$ and $\zeta \zeta^{*}$ channels are presented in Figs. 10(d)-10(h). 


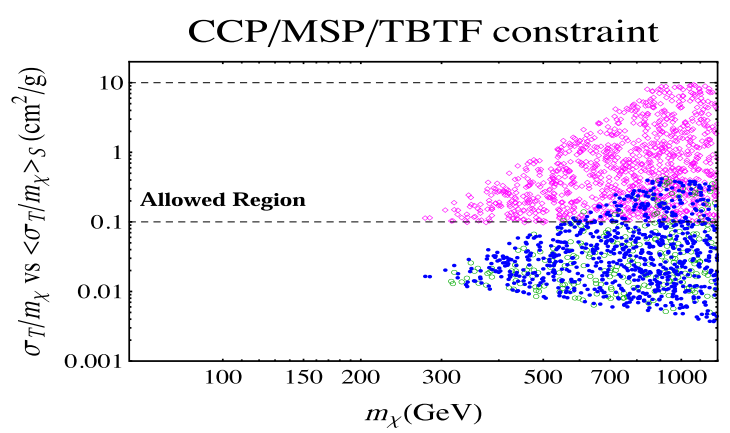

(a)

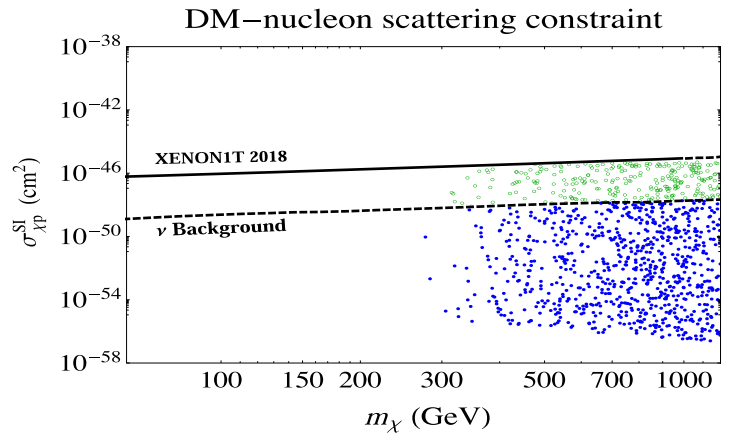

(c)

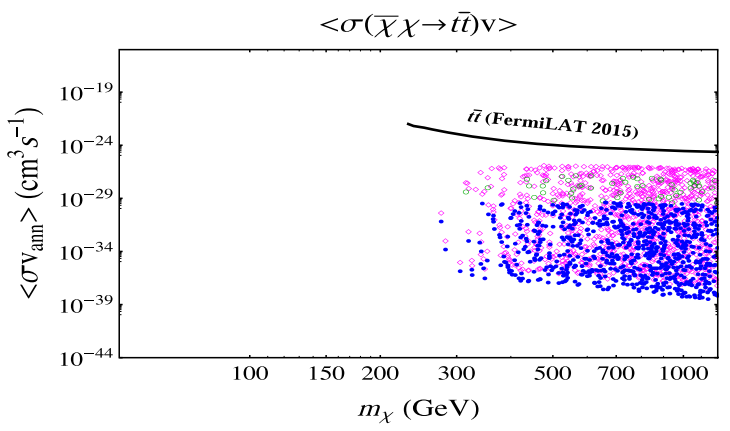

(e)

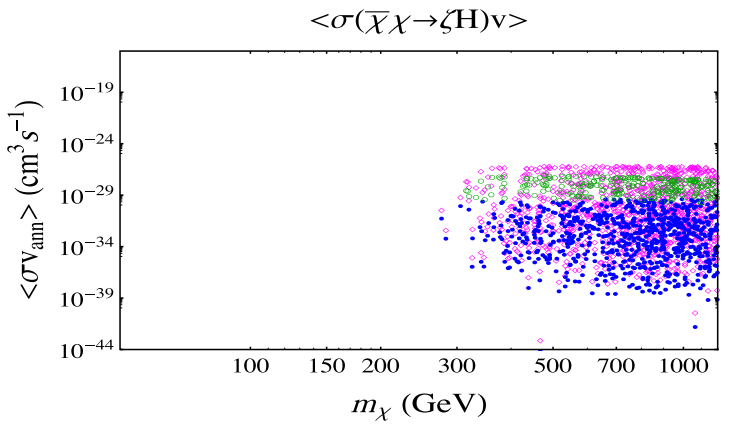

(g)

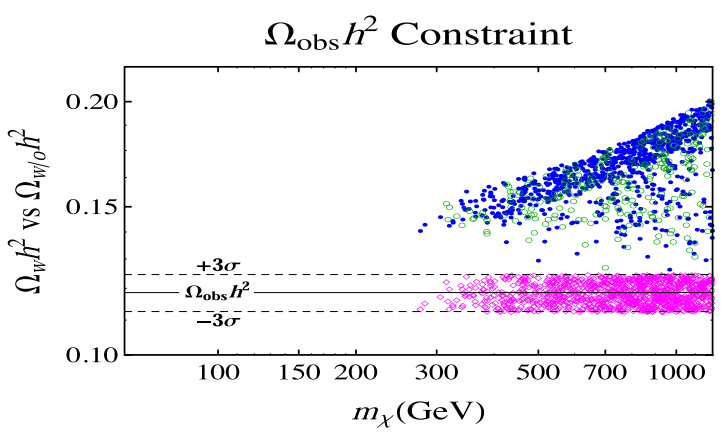

(b)

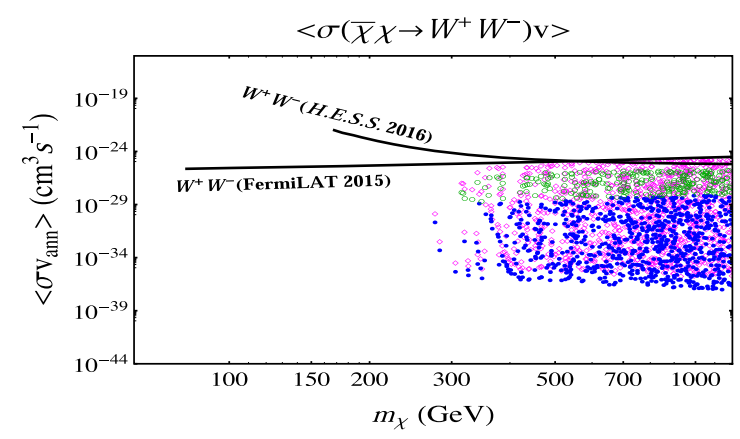

(d)

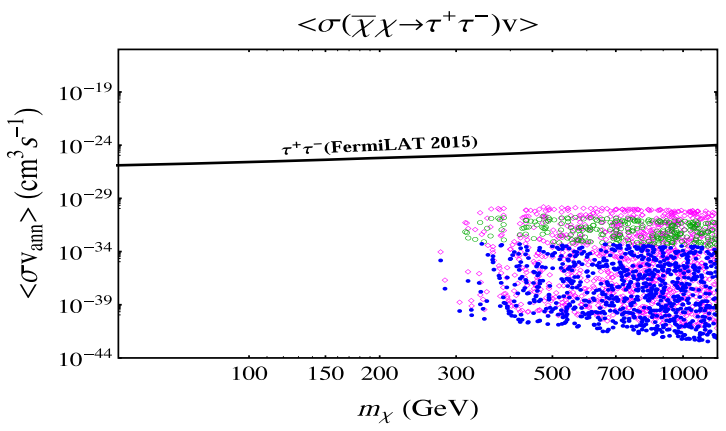

(f)

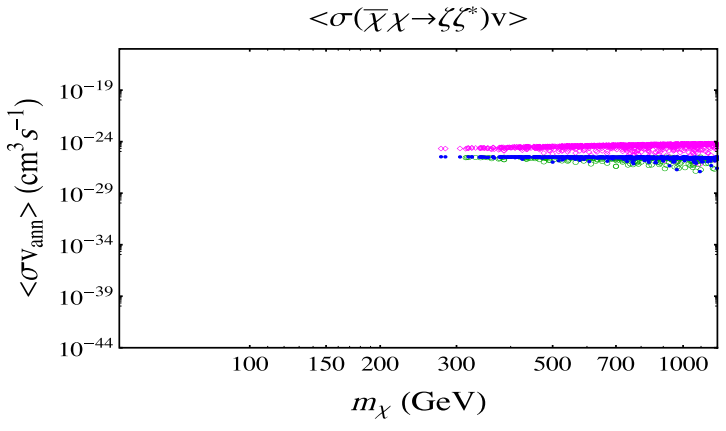

(h)

FIG. 10. Predictions on $\sigma_{T} / m_{\chi}, \Omega h^{2}, \sigma^{S I}$, and $\left\langle\sigma_{\text {ann }} v\right\rangle$ in different channels for all allowed samples. (Circle) and (filled circle) denote the samples which are testable for near future and below the neutrino floor, respectively, by the direct-detection experiment and without consideration of Sommerfeld effect. (Diamond) denotes the values with considering the Sommerfeld effect.

Combining indirect search in Fig. 8 and the small scale requirement, we see that the values of $\left\langle\sigma_{\text {ann }} v\right\rangle$ cannot be too large, and that $\chi \chi \rightarrow b \bar{b}$ and $\tau^{+} \tau^{-}$may not be detectable.
Recall that the dominant $t$-channel cross section for the self-interacting leptonic scalar DM at the tree level is 


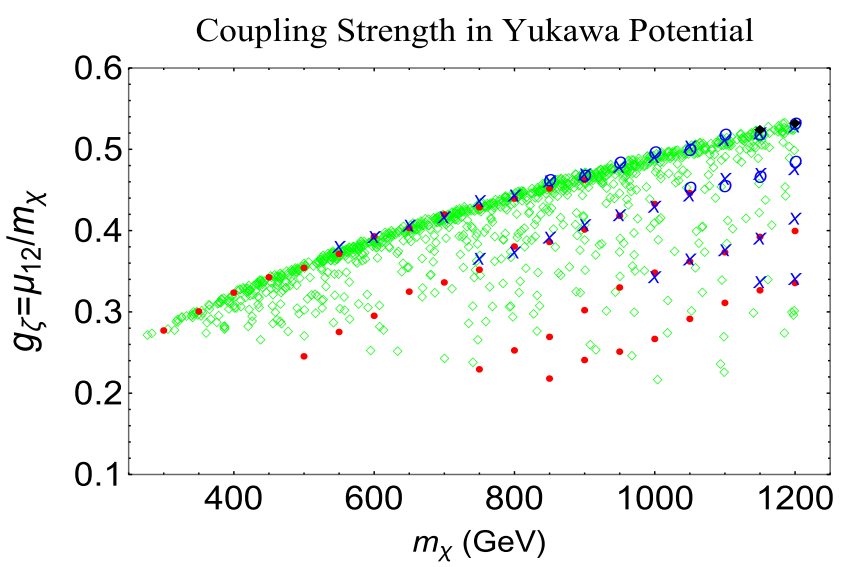

FIG. 11. The dimensionless coupling strength $g_{\zeta} \equiv \mu_{12} / m_{\chi}$ in the Yukawa potential versus DM mass $m_{\chi}$ parametrized by the light mediator mass $m_{\zeta}$ where $m_{\zeta}=0.2 \mathrm{GeV}$ [red (filled circle)], $0.4 \mathrm{GeV}$ [blue (cross)], $0.6 \mathrm{GeV}$ [blue (circle)], and $0.8 \mathrm{GeV}$ [black (diamond)].

$$
\sigma\left(\chi \chi^{*} \rightarrow \chi \chi^{*}\right)=\frac{\mu_{12}^{4}}{4 \pi m_{\zeta}^{4} m_{\chi}^{2}}=\frac{g_{\zeta}^{2} \mu_{12}^{2}}{4 \pi m_{\zeta}^{4}} .
$$

The dimensionless coupling $g_{\zeta} \equiv \mu_{12} / m_{\chi}$ is important to determine the annihilation cross section, and it appears in the Yukawa potential [Eq. (44)] contributing to the Sommerfeld enhancement effects.

The allowed value of $g_{\zeta}$ is modified by non-perturbative Sommerfeld enhancement effects. Figure 11 presents $g_{\zeta}$ as a function of $m_{\chi}$ with several values of $m_{\zeta}=$ $0.2 \mathrm{GeV}$ [red (filled circle)], $0.4 \mathrm{GeV}$ [blue (cross)], $0.6 \mathrm{GeV}$ [blue (circle)], and $0.8 \mathrm{GeV}$ [black (diamond)]. In addition, we consider four sets of parameters from top to bottom: $\left(\lambda_{01}, \lambda_{02}, \lambda_{12}, f_{R R}\right)=\left(10^{-6}, 10^{-6}, 10^{-6}, 10^{-6}\right)$, $\left(0.1,10^{-3}, 0.1,0.0\right),\left(0.2,10^{-3}, 0.2,0.2\right)$ and $\left(0.2,10^{-3}\right.$, $0.2,0.6)$, respectively. All allowed samples consistent with all mentioned constraints are denoted by green " $\diamond "$. We find that $g_{\zeta}$ becomes larger with increasing value in $m_{\zeta}$ or decreasing values in the parameter set $\left(\lambda_{01}, \lambda_{02}, \lambda_{12}, f_{R R}\right)$. Note that for a given set of parameters, $\mu_{12}$ is randomly sampled to satisfy all mentioned constraint. The allowed range of $m_{\zeta}$ also depends on $m_{\chi}$.

From the 1000 allowed samples that are consistent with all mentioned constraints, we can find the favored parameter space from the scanned region. BBN and CMB constraints require that $m_{\zeta}$ should be greater than $200 \mathrm{MeV}$, that leads to the minimal value of $m_{\chi}=276 \mathrm{GeV}$. The maximal value of $m_{\zeta}$ is found to be $814 \mathrm{MeV}$ corresponding to $m_{\chi}=1176 \mathrm{GeV} . \quad \lambda_{1}$ only involves the selfinteraction process of $\chi \chi * \rightarrow \chi \chi^{*}$ in Eq. (67) and its contribution can be ignored even with $\lambda_{1}=\sqrt{4 \pi}$. On the other hand, $\lambda_{2}$ is irrelevant in our calculation. We find that the maximal values of $\lambda_{01}, \lambda_{02}, \lambda_{12}$ and $f_{R R}$ are $0.27,0.01$
0.51 and 1.30 , respectively. The allowed range of $\mu_{12}$ is between 75 and $634 \mathrm{GeV}$.

\section{CONCLUSIONS}

We have adopted a special model that has a leptonic scalar dark matter (LSDM) $(\chi)$ with lepton number $L_{\chi}=1$ and a light scalar mediator $(\zeta)$ with $L_{\zeta}=2$ and three flavors of neutrino $\nu_{R}$ under the assumption of lepton number conservation. In the early Universe, DM thermalizes with SM particles via $H$-portal and $\chi \chi^{*} \rightarrow \zeta \zeta^{*}$ provides an efficient annihilation channel. After DM freezes out, all $\zeta$ decay into $\nu_{R} \nu_{R}$ with a lifetime $\tau_{\zeta} \lesssim 10^{-11}$ (sec) before the onset of $\mathrm{BBN}$, and $\nu_{R}$ decouples from the SM particles at the temperature $T_{f}^{R} \sim m_{\zeta}$. The LSDM $(\chi)$ and the light mediator $(\zeta)$ with lepton number conservation can provide a self-interacting WIMP dark matter that is consistent with astrophysical and cosmological constraints.

In the LSDM model, the $t$ - channel exchange of a light mediator $(\zeta)$ makes the LSDM $(\chi)$ self interacting cross section (SICS) reasonable large. Furthermore, we evaluate the Sommerfeld effects and find significant enhancement for the SICS. That makes the LSDM model suitable to explain the small scale structure of the Universe.

We apply selection requirements for small scale structure, cold dark matter relic density (Planck), direct searches (XENON1T), and indirect detections (Fermi-LAT and H.E.S.S), as well as cosmological constraints on righthanded neutrinos. A randomly selected set of parameters was found with 1000 samples that satisfy all constraints. Large regions of the parameter space in the LSDM model are found to be consistent with astrophysical and cosmological observations and collider Higgs properties. A summary is in the following for the favored ranges of parameters:

(i) $0.2 \mathrm{GeV} \lesssim m_{\zeta} \lesssim 0.814 \mathrm{GeV}$ (BBN, CMB),

(ii) $276 \mathrm{GeV} \lesssim m_{\chi} \lesssim 1176 \mathrm{GeV}$ (implied by $m_{\zeta}$ ),

(iii) $75 \mathrm{GeV} \leq \mu_{12} \leq 634 \mathrm{GeV}$, and

(iv) $\lambda_{02} \leq 10^{-2}$ (Higgs invisible width).

In addition, the upper bound of $\lambda_{01}, \lambda_{02}$ and $\lambda_{12}$ are 0.27 , 0.01 and 0.51 , respectively.

It is interesting that almost all regions of parameter space satisfying astrophysical and cosmological observations lead to a cold dark matter relic density with the most restrictive requirement [8].

$$
\Omega_{\mathrm{c}} h^{2} \pm 3 \sigma=0.120 \pm 0.001
$$

that is

$$
0.117 \lesssim \Omega_{\chi} h^{2} \lesssim 0.123
$$

A more realistic requirement should be 


$$
\Omega_{\chi} h^{2} \lesssim 0.123
$$

That will enlarge the favored parameter space and accommodate more types of dark matter particles.

\section{ACKNOWLEDGMENTS}

We are grateful to Ernest Ma for beneficial discussion. This research was supported by Grants No. MOST 1092811-M-002-530 and No. NTU 108L104019 (G.-G. W.), as well as in part by the U.S. Department of Energy and the University of Oklahoma (C. K.). Y.-L. S. Tsai was funded by the Ministry of Science and Technology Taiwan under Grant No. 109-2112-M-007-022-MY3.

\section{APPENDIX A: SOMMERFELD ENHANCEMENT IN $\chi \chi$ ANNIHILATIONS}

\section{Bethe-Salpeter equation in $\chi \chi \rightarrow \chi \chi$ process}

The Feynman diagram of nonperturbative scattering $\chi\left(p_{1}\right) \chi\left(p_{2}\right) \rightarrow \chi\left(p_{3}^{\prime}\right) \chi\left(p_{4}^{\prime}\right)$ is shown in Fig. 5(a). Note that $p_{3}^{\prime}$ and $p_{4}^{\prime}$ are not necessary on-shell as these two lines will be connected to $\chi \chi$ annihilation diagrams later [see Fig. 5(b)]. Following the standard procedure $[68,75]$, we will derive the Bathe-Salpeter equation for the process of scalar DM scattering via the scalar $H$-exchange repeatedly.

The amputated nonperturbative 4-point vertex function can be written as

$$
i \Gamma\left(p_{3}^{\prime}, p_{4}^{\prime} ; p_{1}, p_{2}\right)=i \tilde{\Gamma}\left(p_{3}^{\prime}, p_{4}^{\prime} ; p_{1}, p_{2}\right)+\int \frac{d^{4} p_{3}^{\prime \prime}}{(2 \pi)^{4}} i \tilde{\Gamma}\left(p_{3}^{\prime}, p_{4}^{\prime} ; p_{3}^{\prime \prime}, p_{4}^{\prime \prime}\right)\left(i D_{F}\left(p_{3}^{\prime \prime}\right)\right) i \Gamma\left(p_{3}^{\prime \prime}, p_{4}^{\prime \prime} ; p_{1}, p_{2}\right)\left(i D_{F}\left(p_{4}^{\prime \prime}\right)\right)
$$

where we have $p_{4}^{\prime \prime}=p_{3}^{\prime}+p_{4}^{\prime}-p_{3}^{\prime \prime}, D_{F}$ is the scalar DM propagator and, the amputated tree-level 4-point vertex function through the $H$-exchange is given by

$$
i \tilde{\Gamma}\left(p_{3}^{\prime}, p_{4}^{\prime} ; p_{3}^{\prime \prime}, p_{4}^{\prime \prime}\right)=-i \lambda_{01}^{2} v^{2} \frac{1}{\left(p_{3}^{\prime \prime}-p_{3}^{\prime}\right)^{2}-m_{H}^{2}} .
$$

With the instantaneous approximation, namely, ignoring the time component of the momentum transfer, the tree-level 4-point vertex function is just the potential $U_{H}\left(\vec{p}_{3}^{\prime \prime}-\vec{p}_{3}^{\prime}\right)$ defined below

$$
\begin{aligned}
i \tilde{\Gamma}\left(p_{3}^{\prime}, p_{4}^{\prime} ; p_{3}^{\prime \prime}, p_{4}^{\prime \prime}\right) & =i \lambda_{01}^{2} v^{2} \frac{1}{\left(\vec{p}_{3}^{\prime \prime}-\vec{p}_{3}^{\prime}\right)^{2}+m_{H}^{2}} \\
& \equiv i U_{H}\left(\vec{p}_{3}^{\prime \prime}-\vec{p}_{3}^{\prime}\right) .
\end{aligned}
$$

To proceed we define two auxiliary functions as follows [68]:

$i \eta\left(p_{3}, p_{4} ; p_{1}, p_{2}\right) \equiv i D_{F}\left(p_{3}\right) i \Gamma\left(p_{3}, p_{4} ; p_{1}, p_{2}\right)\left(i D_{F}\left(p_{4}\right)\right)$, $i \tilde{\chi}\left(p_{3}, p_{4} ; p_{1}, p_{2}\right) \equiv i D_{F}\left(p_{3}\right) i \tilde{\Gamma}\left(p_{3}, p_{4} ; p_{1}, p_{1}\right)\left(i D_{F}\left(p_{4}\right)\right)$,

and Eq. (A1) can be expressed as

$$
\begin{aligned}
& i \eta\left(p_{3}^{\prime}, p_{4}^{\prime} ; p_{1}, p_{2}\right) \\
& \quad=i \tilde{\chi}\left(p_{3}^{\prime}, p_{4}^{\prime} ; p_{1}, p_{2}\right) \\
& \quad+\int \frac{d^{4} p_{3}^{\prime \prime}}{(2 \pi)^{4}} i \tilde{\chi}\left(p_{3}^{\prime}, p_{4}^{\prime} ; p_{3}^{\prime \prime}, p_{4}^{\prime \prime}\right) i \eta\left(p_{3}^{\prime \prime}, p_{4}^{\prime \prime} ; p_{1}, p_{2}\right) .
\end{aligned}
$$

Adding $(2 \pi)^{4} \delta^{4}\left(p_{3}^{\prime \prime}-p_{1}\right)$ to both side of the above equation, and defining

$i \chi\left(p_{3}^{\prime}, p_{4}^{\prime} ; p_{1}, p_{2}\right) \equiv(2 \pi)^{4} \delta^{4}\left(p_{3}^{\prime \prime}-p_{3}^{\prime}\right)+i \eta\left(p_{3}^{\prime}, p_{4}^{\prime} ; p_{1}, p_{2}\right)$,

Eq. (A5) becomes

$$
\begin{aligned}
i \chi\left(p_{3}^{\prime}, p_{4}^{\prime} ; p_{1}, p_{2}\right)= & (2 \pi)^{4} \delta^{4}\left(p_{3}^{\prime \prime}-p_{3}^{\prime}\right) \\
& +\int \frac{d^{4} p_{3}^{\prime \prime}}{(2 \pi)^{4}} i \tilde{\chi}\left(p_{3}^{\prime}, p_{4}^{\prime} ; p_{3}^{\prime \prime}, p_{4}^{\prime \prime}\right) \\
& \times i \chi\left(p_{3}^{\prime \prime}, p_{4}^{\prime \prime} ; p_{1}, p_{2}\right) .
\end{aligned}
$$

In the NR limit, the scalar propagator can be approximately written as

$D_{F}( \pm k)=\frac{1}{2 m_{\chi}} \frac{1}{\left(k_{0}-m_{\chi}\right)-\vec{k}^{2} / 2 m_{\chi}+i \epsilon} \equiv \frac{1}{2 m_{\chi}} g_{\chi}(k)$.

Substitute it into the above equation, we obtain the equation for $\chi$, 


$$
i \chi\left(p_{3}^{\prime}, p_{4}^{\prime} ; p_{1}, p_{2}\right)=(2 \pi)^{4} \delta^{4}\left(p_{3}^{\prime \prime}-p_{3}^{\prime}\right)+\frac{1}{4 m_{\chi}^{2}} g_{\chi}\left(p_{3}^{\prime}\right) g_{\chi}\left(p_{4}^{\prime}\right) \int \frac{d^{4} p_{3}^{\prime \prime}}{(2 \pi)^{4}} U_{H}\left(\vec{p}_{3}^{\prime \prime}-\vec{p}_{3}^{\prime}\right) \chi\left(p_{3}^{\prime \prime}, p_{4}^{\prime \prime} ; p_{1}, p_{2}\right)
$$

In fact, we can drop the redundant variables $p_{1}$ and $p_{2}$ in the above equation. Now we define

$$
\begin{aligned}
p & \equiv\left(p_{3}^{\prime}-p_{4}^{\prime}\right) / 2 \\
P & \equiv\left(p_{3}^{\prime}+p_{4}^{\prime}\right) / 2 \\
\hat{\chi}\left(k_{1}, k_{2}\right) & \equiv \chi\left(k_{1}+k_{2}, k_{1}-k_{2}\right) .
\end{aligned}
$$

Eq. (A9) can be rewritten as

$$
i \hat{\chi}(P, p)=(2 \pi)^{4} \delta^{4}(q-P-p)+\frac{1}{4 m_{\chi}^{2}} g_{\chi}(P+p) g_{\chi}(P-p) \int \frac{d^{4} q}{(2 \pi)^{4}} U_{H}(\vec{q}-\vec{P}-\vec{p}) \hat{\chi}(P, q-P)
$$

Let $q^{\prime}=q-P$ and redefine $q^{\prime}=q$. The above equation becomes

$$
i \hat{\chi}(P, p)=\left(2 \pi^{4}\right) \delta^{4}(q-p)+\frac{1}{4 m_{\chi}^{2}} g_{\chi}(P+p) g_{\chi}(P-p) \int \frac{d^{4} q}{(2 \pi)^{4}} U_{H}(\vec{q}-\vec{p}) \hat{\chi}(P, q) .
$$

Defining the Bathe-Salpeter wave function as

$$
\psi(\vec{q}) \equiv \int \frac{d q_{0}}{2 \pi} i \hat{\chi}(P, q)
$$

and integrating with respect to $p_{0}$ on both sides of Eq. (A12), we have

$$
\psi(\vec{p})=(2 \pi)^{3} \delta(\vec{q}-\vec{p})+\frac{1}{2 \pi i} \frac{1}{4 m_{\chi}^{2}} \int d p_{0} g_{\chi}(P+p) g_{\chi}(P-p) \int \frac{d^{3} \vec{q}}{(2 \pi)^{3}} U_{H}(\vec{q}-\vec{p}) \psi(\vec{q}) .
$$

By taking $p_{3}^{\prime}=\left(E_{3}^{\prime}, \vec{p}\right), p_{4}^{\prime}=\left(E_{4}^{\prime},-\vec{p}\right), p_{3}^{\prime \prime}=\left(E_{3}^{\prime \prime}, \vec{p}\right)$, and $p_{4}^{\prime}=\left(E_{4}^{\prime \prime},-\vec{p}\right)$ in the center of mass frame, we have $p \equiv$ $(\epsilon, \vec{p})=\left(\left(E_{3}^{\prime}-E_{4}^{\prime}\right) / 2, \vec{p}\right)$ and $P \simeq\left(m_{\chi}+E / 2, \overrightarrow{0}\right)$ and the total kinetic energy $E=\mu v^{2} / 2$ where $\mu=m_{\chi} / 2$ is the reduce mass of $\chi \chi$ system and the relative velocity $v=v_{\text {lab }}$ defined in Eq. (28). Using the residue theorem, we integrate over $p_{0}=\epsilon$ in Eq. (A14), and obtain

$$
\psi(\vec{p})=(2 \pi)^{3} \delta(\vec{q}-\vec{p})+\frac{1}{E-\frac{\vec{p}^{2}}{m_{\chi}}} \int \frac{d^{3} \vec{q}}{(2 \pi)^{3}} V_{H}(\vec{q}-\vec{p}) \psi(\vec{q}), \quad V_{H}(\vec{q}) \equiv-\frac{1}{4 m_{\chi}^{2}} U_{H}(\vec{q}),
$$

The above equation is simply the Bathe-Salpeter equation in the momentum space representation. By taking the Fourier transformation, we have

$$
\int \frac{d^{3} \vec{p}}{(2 \pi)^{3}} e^{-i \vec{p} \cdot \vec{r}}\left(\frac{\vec{p}^{2}}{m_{\chi}}-E\right)\left[\psi(\vec{p})+(2 \pi)^{3} \delta(\vec{q}-\vec{p})\right]+\int \frac{d^{3} \vec{p}}{(2 \pi)^{3}} e^{-i \vec{p} \cdot \vec{r}} \int \frac{d^{3} \vec{q}}{(2 \pi)^{3}} V_{H}(\vec{q}-\vec{p}) \psi(\vec{q})=0
$$

After simplification, we obtain the Bathe-Salpeter equation in the position representation:

$$
-\frac{1}{2 \mu} \nabla^{2} \psi(\vec{r})+V_{H}(r) \psi(\vec{r})=E \psi(\vec{r}), \quad V_{H}(r)=-\alpha_{H} \frac{e^{-m_{H} r}}{r}
$$

where we see the potential is Yukawa-type with the corresponding fine structure constant $\alpha_{H}=g_{H}^{2} / 4 \pi$ and the dimensionless coupling strength $g_{H}=\lambda_{01} v / 2 m_{\chi}$. 


\section{2. s-wave Sommerfeld factor in $\chi \chi \rightarrow \nu_{R} \nu_{R}$ process}

From Fig. 5(b), the Sommerfeld enhanced amplitude $i A_{S}$ of $\chi \chi \rightarrow \nu_{R} \nu_{R}$ annihilation process can be expressed as

$$
i A_{S}\left(p_{3}, p_{4}, p_{1}, p_{2}\right)=i A\left(p_{3}, p_{4}, p_{1}, p_{2}\right)+\int \frac{d^{4} p_{3}^{\prime}}{(2 \pi)^{4}} i A\left(p_{3}, p_{4} ; p_{3}^{\prime}, p_{4}^{\prime}\right)\left(i D_{F}\left(p_{3}^{\prime}\right)\right) i \Gamma\left(p_{3}^{\prime}, p_{4}^{\prime} ; p_{1}, p_{2}\right)\left(i D_{F}\left(p_{4}^{\prime}\right)\right)
$$

where $i A$ is the amplitude of the process at tree level. With the help of Eq. (A4), we have

$$
\begin{aligned}
i A_{S} & \left(p_{3}, p_{4} ; p_{1}, p_{2}\right) \\
& =\int \frac{d^{4} p_{3}^{\prime}}{(2 \pi)^{4}} i A\left(p_{3}, p_{4} ; p_{3}^{\prime}, p_{4}^{\prime}\right) i \chi\left(p_{3}^{\prime}, p_{4}^{\prime} ; p_{1}, p_{2}\right) .
\end{aligned}
$$

For the s-wave rescattering, the amplitude $A\left(p_{3}, p_{4} ; p_{3}^{\prime}, p_{4}^{\prime}\right)$ is independent of momentum and hence, the above equation becomes

$$
\begin{aligned}
& i A_{S}\left(p_{3}, p_{4}, ; p_{1}, p_{2}\right) \\
& \quad=i A\left(p_{3}, p_{4} ; p_{1}, p_{2}\right) \int \frac{d^{3} \vec{p}}{(2 \pi)^{4}} \int \frac{d p_{0}}{(2 \pi)} i \hat{\chi}(P, p) \\
& \quad=i A\left(p_{3}, p_{4} ; p_{1}, p_{2}\right) \psi_{l=0}(\vec{r}=0)
\end{aligned}
$$

Hence we have

$$
\left.\left|A_{S}\left(p_{3}, p_{4}, ; p_{1}, p_{2}\right)\right|^{2}=\mid A_{(} p_{3}, p_{4}, ; p_{1}, p_{2}\right)\left.\right|^{2} S
$$

where $S=\left|\psi_{l=0}(\vec{r}=0)\right|^{2}$ is the so-called s-wave Sommerfeld factor and the wave function $\psi(\vec{r})$ satisfies the Bathe-Salpeter equation in Eq. (A17).

\section{Solving $\psi(\vec{r}=0)$ numerically}

Let us consider the general case. As we know that two DM particles form a bound $\chi \chi$ or $\chi \chi^{*}$ state before annihilation. This two-particle wave function $\psi(\vec{r})$ satisfies the following Schrödinger equation [see in Eq. (43)]

$-\frac{1}{2 \mu} \nabla^{2} \psi(\vec{r})+V(\vec{r}) \psi(\vec{r})=E \psi(\vec{r})=\frac{1}{2} \mu v^{2} \psi(\vec{r})$.

The separation of variables give us the radial Schrödinger equation

$\left[-\frac{1}{2 \mu} \frac{1}{r^{2}} \frac{d}{d r}\left(r^{2} \frac{d}{d r}\right)+V(r)+\frac{l(l+1)}{2 \mu r^{2}}\right] R_{l}(r)=E R_{l}(r)$.

Here we follow $[68,76]$ to solve for $\psi(\vec{r}=0)$ numerically. From the scattering theory, the radial wave function has the following asymptotic form [77]:

$$
R_{l}(r) \rightarrow e^{i \delta_{l}} \frac{\sin \left(p r-l \pi / 2+\delta_{l}\right)}{p r}
$$

where $\delta_{l}$ is the phase shift corresponding to the partial wave with angular momentum quantum number $l$. Defining $\Phi_{l}$ by $R_{l}(\rho)=N \rho^{l} \Phi_{l}(r)$ with $\rho=p r$, and normalization constant $N$ to be determined later, Eq. (A23) becomes

$$
\Phi_{l}^{\prime \prime}+\frac{2(l+1)}{\rho} \Phi_{l}^{\prime}+\left(-\frac{2}{p v} V(r)+1\right) \Phi_{l}=0,
$$

where the initial conditions are taken to be [76]

$$
\Phi_{l}(0)=1, \quad \Phi_{l}^{\prime}(0)=\left.\frac{\rho V(r)}{p v(l+1)}\right|_{\rho \rightarrow 0} \Phi_{l}(0),
$$

for a regular solution. We now concentrate on the $l=0$ case. As one can see by taking $\rho \gg 1$, in the case that $|\rho V(r)| \ll 1$, the differential equation and its solution become

$$
\Phi_{0}^{\prime \prime}+\frac{2}{\rho} \Phi_{0}^{\prime}+\left.\Phi_{0}\right|_{\rho \gg 1}=0, \quad \Phi_{0}(\rho) \rightarrow C \frac{\sin \left(\rho+\delta_{0}\right)}{\rho},
$$

with $C$ a real number. The above $\Phi_{0}$ is to be compared to $R_{0}(r) \rightarrow e^{i \delta_{0}} \sin \left(\rho+\delta_{0}\right) / \rho$ [see Eq. (A24)], as $\rho \gg 1$. To work out the normalization $N$, it is useful noting, in the $\rho \gg 1$ region,

$$
\Phi_{0}(\rho-\pi / 2) \rightarrow-C \frac{\cos \left(\rho+\delta_{0}\right)}{\rho-\pi / 2},
$$

which can be used with $\Phi_{0}(\rho)$ to construct

$\kappa \equiv \lim _{\rho \rightarrow \infty} e^{i \rho}\left[-i \rho \Phi_{0}(\rho)-(\rho-\pi / 2) \Phi_{0}(\rho-\pi / 2)\right]=C e^{-i \delta_{0}}$.

Consequently, we see that $R_{0}(r)$ can be obtained as

$$
R_{0}(r)=\kappa^{-1} \Phi_{0}(\rho)
$$

since it satisfies the Schrödinger equation and has the correct asymptotic behavior. Finally, we have 


$$
\begin{aligned}
\psi(\vec{r}=0) & =\kappa^{-1} \Phi_{0}(0) \\
& =\kappa^{-1}=\lim _{\rho \rightarrow \infty} \frac{e^{-i \rho}}{-i \rho \Phi_{0}(\rho)-(\rho-\pi / 2) \Phi_{0}(\rho-\pi / 2)} .
\end{aligned}
$$

Note that the phase of $\psi(\vec{r}=0)$ is just $\delta_{0}$ [see Eq. (A29)].

Now we are ready to do the numerical calculation. For a scalar DM $\chi$ with a scalar mediator $X$, we have the Yukawatype potential:

$$
V_{X}(r)=-\alpha_{X} \frac{e^{-m_{X} r}}{r}
$$

where $\alpha_{X}=g_{X}^{\prime 2} /\left(16 \pi m_{\chi}^{2}\right)$ with the dimensionful coupling strength $g_{X}^{\prime}=\lambda_{01} \mathrm{v}$, namely, the dimensionless coupling strength $g_{X}=g_{X}^{\prime} / 2 m_{\chi}$. Hence we need to solve the following differential equation:

$$
\Phi^{\prime \prime}(\rho)+\frac{2}{x} \Phi^{\prime}(\rho)+\left(1-\frac{2 a_{X} e^{-b_{X} \rho}}{\rho}\right) \Phi(\rho)=0,
$$

with the boundary conditions:

$$
\Phi(\rho=0)=1, \quad \Phi^{\prime}(\rho=0)=-a_{X} .
$$

In the above $a_{X}=\alpha_{X}$ and $b_{X}=2 m_{X} / m_{\chi} v$. We find that it is enough to take $\rho \simeq 200$ to obtain the limit in Eq. (A31).
[1] F. Zwicky, Helv. Phys. Acta 6, 110 (1933).

[2] V. C. Rubin and W. K. Ford, Jr., Astrophys. J. 159, 379 (1970).

[3] K. G. Begeman, A. H. Broeils, and R. H. Sanders, Mon. Not. R. Astron. Soc. 249, 523 (1991).

[4] S. M. Carroll, Nat. Phys. 2, 653 (2006).

[5] D. Clowe, M. Bradac, A. H. Gonzalez, M. Markevitch, S. W. Randall, C. Jones, and D. Zaritsky, Astrophys. J. 648, L109 (2006).

[6] D. N. Spergel et al. (WMAP Collaboration), Astrophys. J. Suppl. Ser. 148, 175 (2003).

[7] M. Tegmark et al. (SDSS Collaboration), Phys. Rev. D 69 , 103501 (2004).

[8] N. Aghanim et al. (Planck Collaboration), Astron. Astrophys. 641, A6 (2020).

[9] M. Colless et al. (2DFGRS Collaboration), Mon. Not. R. Astron. Soc. 328, 1039 (2001).

[10] V. Springel, C. S. Frenk, and S. D. M. White, Nature (London) 440, 1137 (2006).

[11] B. Moore, Nature (London) 370, 629 (1994).

[12] B. Moore, S. Ghigna, F. Governato, G. Lake, T. R. Quinn, J. Stadel, and P. Tozzi, Astrophys. J. 524, L19 (1999).

[13] M. Boylan-Kolchin, J. S. Bullock, and M. Kaplinghat, Mon. Not. R. Astron. Soc. 415, L40 (2011).

[14] M. Boylan-Kolchin, J.S. Bullock, and M. Kaplinghat, Mon. Not. R. Astron. Soc. 422, 1203 (2012).

[15] D. N. Spergel and P. J. Steinhardt, Phys. Rev. Lett. 84, 3760 (2000).

[16] M. Rocha, A. H. G. Peter, J. S. Bullock, M. Kaplinghat, S. Garrison-Kimmel, J. Onorbe, and L. A. Moustakas, Mon. Not. R. Astron. Soc. 430, 81 (2013).

[17] O. D. Elbert, J. S. Bullock, S. Garrison-Kimmel, M. Rocha, J. Oñorbe, and A. H. G. Peter, Mon. Not. R. Astron. Soc. 453, 29 (2015).

[18] O. Adriani et al. (PAMELA Collaboration), Nature (London) 458, 607 (2009); Phys. Rev. Lett. 111, 081102 (2013).
[19] M. Aguilar et al. (AMS Collaboration), Phys. Rev. Lett. 110, 141102 (2013); L. Accardo et al. (AMS Collaboration), Phys. Rev. Lett. 113, 121101 (2014); M. Aguilar et al. (AMS Collaboration), Phys. Rev. Lett. 113, 121102 (2014).

[20] J. Chang et al., Nature (London) 456, 362 (2008).

[21] A. A. Abdo et al. (Fermi LAT Collaboration), Phys. Rev. Lett. 102, 181101 (2009).

[22] J. L. Feng, M. Kaplinghat, and H. B. Yu, Phys. Rev. Lett. 104, 151301 (2010).

[23] M. Kaplinghat, T. Linden, and H. B. Yu, Phys. Rev. Lett. 114, 211303 (2015).

[24] E. Ma, Mod. Phys. Lett. A 33, 1850226 (2018).

[25] T. Bringmann, F. Kahlhoefer, K. Schmidt-Hoberg, and P. Walia, Phys. Rev. Lett. 118, 141802 (2017).

[26] E. Aprile et al. (XENON Collaboration), Phys. Rev. Lett. 121, 111302 (2018).

[27] M. Tanabashi et al. (Particle Data Group), Phys. Rev. D 98 , 030001 (2018).

[28] M. Ackermann et al. (Fermi-LAT Collaboration), Phys. Rev. Lett. 115, 231301 (2015).

[29] A. Albert et al. (Fermi-LAT and DES Collaborations), Astrophys. J. 834, 110 (2017).

[30] H. Abdallah et al. (H.E.S.S. Collaboration), Phys. Rev. Lett. 117, 111301 (2016).

[31] C. Bonilla, E. Ma, E. Peinado, and J. W. F. Valle, Phys. Lett. B 762, 214 (2016).

[32] E. Ma and O. Popov, Phys. Lett. B 764, 142 (2017).

[33] E. Ma and U. Sarkar, Phys. Lett. B 776, 54 (2018).

[34] E. Aprile et al. (XENON Collaboration), Phys. Rev. D 102, 072004 (2020).

[35] T. P. Cheng, Phys. Rev. D 38, 2869 (1988).

[36] H. Y. Cheng, Phys. Lett. B 219, 347 (1989).

[37] J. Gasser, H. Leutwyler, and M. E. Sainio, Phys. Lett. B 253, 252 (1991).

[38] J. M. Alarcon, J. Martin Camalich, and J. A. Oller, Phys. Rev. D 85, 051503 (2012).

[39] J. M. Alarcon, L. S. Geng, J. Martin Camalich, and J. A. Oller, Phys. Lett. B 730, 342 (2014). 
[40] H. Y. Cheng and C. W. Chiang, J. High Energy Phys. 07 (2012) 009.

[41] M. A. Shifman, A. I. Vainshtein, and V. I. Zakharov, Phys. Lett. B 78, 443 (1978).

[42] G. Jungman, M. Kamionkowski, and K. Griest, Phys. Rep. 267, 195 (1996).

[43] J. Menendez, D. Gazit, and A. Schwenk, Phys. Rev. D 86, 103511 (2012).

[44] C. K. Chua and G. G. Wong, Phys. Rev. D 94, 035002 (2016).

[45] J. D. Lewin and P. F. Smith, Astropart. Phys. 6, 87 (1996).

[46] L. Vietze, P. Klos, J. Menendez, W. C. Haxton, and A. Schwenk, Phys. Rev. D 91, 043520 (2015).

[47] J. Billard, L. Strigari, and E. Figueroa-Feliciano, Phys. Rev. D 89, 023524 (2014).

[48] B. Cabrera, L. M. Krauss, and F. Wilczek, Phys. Rev. Lett. 55, 25 (1985).

[49] J. Monroe and P. Fisher, Phys. Rev. D 76, 033007 (2007).

[50] E. W. Kolb and M. S. Turner, Front. Phys. 69, 1 (1990).

[51] T. S. Coleman and M. Roos, Phys. Rev. D 68, 027702 (2003).

[52] L. Rinchiuso (H. E. S. S. Collaboration), EPJ Web Conf. 209, 01023 (2019).

[53] A. Albert et al. (HAWC Collaboration), Astrophys. J. 853, 154 (2018).

[54] M. L. Ahnen et al. (MAGIC Collaboration), J. Cosmol. Astropart. Phys. 03 (2018) 009.

[55] S. Archambault et al. (VERITAS Collaboration), Phys. Rev. D 95, 082001 (2017).

[56] A. Albert et al. (ANTARES Collaboration), Phys. Lett. B 805, 135439 (2020).

[57] A. Albert et al. (ANTARES and IceCube Collaborations), Phys. Rev. D 102, 082002 (2020).

[58] C. S. Kochanek, Astrophys. J. 457, 228 (1996).

[59] F. Ferrer and D. R. Hunter, J. Cosmol. Astropart. Phys. 09 (2013) 005.
[60] J. Hisano, S. Matsumoto, and M. M. Nojiri, Phys. Rev. Lett. 92, 031303 (2004).

[61] J. Hisano, S. Matsumoto, M. M. Nojiri, and O. Saito, Phys. Rev. D 71, 063528 (2005).

[62] J. Hisano, S. Matsumoto, M. Nagai, O. Saito, and M. Senami, Phys. Lett. B 646, 34 (2007).

[63] N. Arkani-Hamed, D. P. Finkbeiner, T. R. Slatyer, and N. Weiner, Phys. Rev. D 79, 015014 (2009).

[64] E. J. Chun, J. C. Park, and S. Scopel, J. Cosmol. Astropart. Phys. 12 (2012) 022.

[65] E. J. Chun and J. C. Park, Phys. Lett. B 750, 372 (2015).

[66] E. J. Chun, S. Jung, and J. C. Park, J. High Energy Phys. 01 (2017) 009.

[67] S. Cassel, J. Phys. G 37, 105009 (2010).

[68] C. K. Chua and G. G. Wong, Phys. Rev. D 96, 115006 (2017).

[69] J. L. Feng, M. Kaplinghat, and H.-B. Yu, Phys. Rev. D 82, 083525 (2010); T. R. Slatyer, J. Cosmol. Astropart. Phys. 02 (2010) 028.

[70] L. D. Landau and E. M. Lifshitz, Quantum Mechanics: Non-Relativistic Theory, Course of Theoretical Physics III, 3rd ed. (Pergamon Press, New York, 1977).

[71] N. N. Lebedev, Special Functions \& Their Applications, Revised ed., translated by R. Silverman, Dover Books on Mathematics (Dover Publications, New York, 1972), pp. 15-261.

[72] G. Mangano, G. Miele, S. Pastor, T. Pinto, O. Pisanti, and P. D. Serpico, Nucl. Phys. B729, 221 (2005).

[73] F. Iocco, G. Mangano, G. Miele, O. Pisanti, and P. D. Serpico, Phys. Rep. 472, 1 (2009).

[74] J. Zhang and S. Zhou, Nucl. Phys. B903, 211 (2016).

[75] E. M. Lifshitz and L.P. Pitaevskii, Relativistic Quantum Theory, A Course of Theoretical Physics Vol. 4 (Pergamon Press, New York, 1973).

[76] R. Iengo, J. High Energy Phys. 05 (2009) 024.

[77] J. J. Sakurai and J. Napolitano, Modern Quantum Physics (Addison-Wesley, Boston, MA, 2011). 